Vietnam Journal of Mechanics, VAST, Vol.40, No. 4 (2018), pp. 325-348

DOI: https://doi.org/10.15625/0866-7136/13498

\title{
SECOND ORDER HOMOGENIZATION OF QUASI-PERIODIC STRUCTURES
}

\author{
Duc Trung Le $\mathbf{e}^{1, *}$, Jean-Jacques Marigo ${ }^{2}$ \\ ${ }^{1}$ Sorbonne Universités, UPMC Univ Paris 06, Institut d'Alembert, Paris, France \\ ${ }^{2}$ Laboratoire de Mécanique du Solide, Ecole Polytechnique, Palaiseau, France \\ *E-mail: ductrung.le@dalembert.upmc.fr \\ Received Frebuary 07, 2018
}

\begin{abstract}
The paper develops a general framework to derive the effective properties of quasi-periodic elastic medium. By using the asymptotic expansion method, the solution is expanded to the second order by solving a sequence of minimization problems. The effective stiffness tensors fields entering in the expression of the macroscopic energy are obtained by solving several families of microscopic problems posed on the unit cell and which bring into play only the microstructure. As an illustrative example, we consider an anti-plane elastic case of a heterogeneous cylinder made of a bi-layer laminate and submitted to the gravity. The unit cell being one-dimensional, all the associated elementary problems can be solved in a closed form and one shows that the effective energy of the medium expanded up to the second order depends not only on the strain gradient, but also on the gradient of the volume fraction $\theta$ characterizing the repartition of the two materials in the laminate.
\end{abstract}

Keywords: homogenization, quasi periodic, strain gradient theories, asymptotic expansions.

\section{INTRODUCTION}

The aim of the homogenization theory is to derive the macroscopic behavior of a system which is heterogeneous at the microscopic level. Homogenization has first been developed for periodic structures by using two-scale asymptotic expansions. The main assumption which justifies the scale separation is that the size of the period is small compared to the size of the medium, their ratio being denoted by $\epsilon$. From the mathematical foundations of the homogenization theory developed by Bensoussan et al. [1]; Murat and Tartar [2]; Allaire [3], numerous works have been devoted to the effective behavior of materials in various applications like composite materials (e.g. Dumontet [4]; Francfort and Murat [5]; Abdelmoula and Marigo [6]), heat diffusion (e.g. Marchenko and Khruslov [7]) or porous media (e.g. Hornung [8]).

The method is based on the construction of a "two-scale" asymptotic expansion of the solution with respect to the small parameter $\epsilon$. If one considers the first order only,

(C) 2018 Vietnam Academy of Science and Technology 
one obtains the classical homogenization theory. But in some applications it is necessary to use higher-order terms of the expansion in order to obtain a better approximation of the solution. The role of these higher-order terms in the asymptotic expansion has been investigated in the case of periodic media by Gambin and Kroner [9], and Boutin [10]. But in many cases, this assumption of perfect periodicity of the heterogeneities is no more valid and must be weakened by considering quasi-periodic media where the coefficients characterizing the behavior of the materials depends both on the macroscopic and microscopic scales. Very few works have been devoted to such media, especially when higher order terms are concerned. For example, the first-order homogenized solution of quasi-periodic structures have been studied by Andrianov et al. [11]. In Su et al. [12], authors propose to construct the second-order solution by adding a series of corrector terms, which are calculated from the first-order solution, but the homogenized equilibrium equation remains the first order equation. Similar ideas can be found in the nonperiodic homogenization of the seismic wave equation presented by Guillot et al. [13], Capdeville et al. [14]. In those papers, the authors seek for a "partial first-order solution" which is corrected from the order 0 solution with the first-order corrector, thus it is not a complete order 1 solution.

The aim of the present work is to provide a higher order macroscopic behavior of a quasi-periodic medium. Specifically, the paper is organized as follows: in Section 1, we set the problem and describe the variational-asymptotic procedure for constructing the second-order macroscopic elastic energy of the quasi-periodic medium. In Section 3, the method is developed in the general case, whereas Section 4 is devoted to the particular case of a quasi periodic bi-layered laminate in an anti-plane situation which allows us to obtain all the effective stiffness tensors in a closed form.

Throughout the paper, we use the following notation. The summation convention on repeated indices is implicitly adopted. The vectors and second order tensors are indicated by boldface letters, like $\mathbf{u}$ and $\sigma$ for the displacement field and the stress field. Their components are denoted by italic letters, like $u_{i}$ and $\sigma_{i j}$. The fourth order tensors as well as their components are indicated by a sans serif letter, like $A$ or $\mathrm{A}_{i j k l}$ for the stiffness tensor. Such tensors are considered as linear maps applying on vectors or second order tensors and the application is denoted without dots, like $A \varepsilon$ whose $i j$-component is $\mathrm{A}_{i j k l} \varepsilon_{k l}$. The inner product between two vectors or two tensors of the same order is indicated by a dot, like $\mathbf{a} \cdot \mathbf{b}$ which stands for $a_{i} b_{i}$ or $\sigma \cdot \varepsilon$ for $\sigma_{i j} \varepsilon_{i j}$. The symbol $\otimes$ denotes the tensor product and $\odot$ its symmetrized, i.e. $2 \mathbf{e}_{1} \odot \mathbf{e}_{2}=\mathbf{e}_{1} \otimes \mathbf{e}_{2}+\mathbf{e}_{2} \otimes \mathbf{e}_{1}$.

As far as the regularity of the fields is concerned, we use the following notation: (i) $\mathcal{D}(\Omega)$ denotes the set of infinitely differentiable (scalar) functions with compact support in the open set $\Omega$ of $\mathbb{R}^{n}$; (ii) $H^{1}\left(\Omega, \mathbb{R}^{m}\right)$ is the set of vector fields defined on $\Omega$ and with values in $\mathbb{R}^{m}$, which are square integrable and whose first derivative is also square integrable; (ii) $H_{0}^{1}\left(\Omega ; \mathbb{R}^{m}\right)$ is the set of vector fields which are in $H^{1}\left(\Omega ; \mathbb{R}^{m}\right)$ and whose trace on the boundary $\partial \Omega$ vanishes; (iv) $H_{\#}^{1}\left(Y ; \mathbb{R}^{m}\right)$ is the set of vector fields which are $Y$-periodic and which belong to $H^{1}\left(Y ; \mathbb{R}^{m}\right)$.

As we make ample use of multiple scaling techniques, we adopt related notation. For instance, $\mathbf{x}=\left(x_{1}, x_{2}, x_{3}\right)$ always denotes a macroscopic coordinate while $\mathbf{y}=\left(y_{1}, y_{2}, y_{3}\right)$ 
will represent a microscopic one. When a spatial (scalar, vectorial or tensorial) field depends both on $\mathbf{x}$ and $\mathbf{y}$, the partial derivative with respect to one of these coordinates appears explicitly as an index: for example, $\partial_{x_{i}} \mathbf{u}(\mathbf{x}, \mathbf{y})$ and $\partial_{y_{i}} \mathbf{u}(\mathbf{x}, \mathbf{y})$ denote respectively the partial derivative of $\mathbf{u}$ with respect to $x_{i}$ and $y_{i}, \operatorname{div}_{\mathbf{x}} \sigma$ and $\varepsilon_{\mathbf{x}}(\mathbf{v})$ denote respectively the divergence of the stress tensor field $\sigma$ and the symmetrized gradient of the vector field $\mathbf{v}$ with respect to $\mathbf{x}$, while $\operatorname{div}_{\mathbf{y}} \sigma$ and $\varepsilon_{\mathbf{y}}(\mathbf{v})$ are the corresponding derivatives with respect to $\mathbf{y}$

$$
\begin{gathered}
\operatorname{div}_{\mathbf{x}} \sigma:=\partial_{x_{j}} \sigma_{i j} \mathbf{e}_{i}, \quad \operatorname{div}_{\mathbf{y}} \sigma:=\partial_{y_{j}} \sigma_{i j} \mathbf{e}_{i}, \\
\varepsilon_{\mathbf{x} i j}(\mathbf{v}):=\frac{1}{2}\left(\partial_{x_{i}} v_{j}+\partial_{x_{j}} v_{i}\right), \quad \varepsilon_{\mathbf{y} i j}(\mathbf{v}):=\frac{1}{2}\left(\partial_{y_{j}} v_{i}+\partial_{y_{j}} v_{j}\right),
\end{gathered}
$$

The mean value of a $Y$-periodic field $\varphi$ is denoted $\langle\varphi\rangle$

$$
\langle\varphi\rangle=: \frac{1}{Y} \int_{Y} \varphi(\mathbf{y}) \mathrm{d} \mathbf{y} .
$$

\section{GOVERNING EQUATIONS}

Let us consider a heterogeneous three-dimensional body $\Omega$ which contains a microstructure involving the micro-cell $Y^{\epsilon}$. We denote $A^{\epsilon}$ the fourth-order elasticity tensor whose components $A_{i j k l}^{\epsilon}$ are assumed to depend quasi periodically on the global coordinates $\mathbf{x}=\left(x_{1}, x_{2}, x_{3}\right)$ in a sense which will be detailed later, see (6). At each point $\mathbf{x}$, the tensor $\mathrm{A}^{\epsilon}(x)$ is symmetric

$$
\mathrm{A}_{i j k l}^{\epsilon}(\mathbf{x})=\mathrm{A}_{j i k l}^{\epsilon}(\mathbf{x})=\mathrm{A}_{j i k l}^{\epsilon}(\mathbf{x}), \quad \forall i, j, k, l
$$

and positive definite in the sense that

$$
\mathrm{A}^{\epsilon}(\mathbf{x}) \boldsymbol{\varepsilon} \cdot \varepsilon \geq 0,
$$

for any symmetric tensor $\varepsilon$, with an equality only if $\varepsilon=0$.

The body is subjected to body forces $\mathbf{f}(\mathbf{x})=\left(f_{i}(\mathbf{x})\right)$, which are assumed to be independent of the microstructure, i.e. $\mathbf{f}$ does not depend on $\epsilon$. These forces $\mathbf{f}$ induce a deformation of the medium, characterized by the displacement field $\mathbf{u}^{\epsilon}(\mathbf{x})=\left(u_{i}^{\epsilon}(\mathbf{x})\right)$. As far as the boundary conditions are concerned, to simplify the presentation, we will assume that all the boundary of the body is clamped and hence that the displacement must satisfy the homogeneous Dirichlet boundary condition

$$
\mathbf{u}^{\epsilon}=\mathbf{0} \quad \text { on } \quad \partial \Omega .
$$

Accordingly, the problem characterizing the equilibrium of the body reads as

$$
\begin{cases}\operatorname{div}_{\mathbf{x}}\left(A^{\epsilon} \mathcal{E}_{\mathbf{x}}\left(\mathbf{u}^{\epsilon}\right)\right)+\mathbf{f}=\mathbf{0}, & \text { in } \Omega \\ \mathbf{u}^{\epsilon}=0, & \text { on } \partial \Omega\end{cases}
$$

Here $\varepsilon_{\mathbf{x}}(\mathbf{u})$ is the linearized strain tensor associated with the displacement $\mathbf{u}$

$$
\varepsilon_{\mathbf{x} k l}(\mathbf{u})=\frac{1}{2}\left(\partial_{x_{l}} u_{k}+\partial_{x_{k}} u_{l}\right)
$$

and the stress tensor $\sigma^{\epsilon}$ at equilibrium is given by

$$
\sigma^{\epsilon}=A^{\epsilon} \varepsilon_{\mathbf{x}}\left(\mathbf{u}^{\epsilon}\right) .
$$


The problem (2) admits the following equivalent variational formulation

Variational formulation of the true problem. Find $\mathbf{u}^{\epsilon} \in H_{0}^{1}\left(\Omega ; \mathbb{R}^{3}\right)$ which minimizes the energy functional $J^{\epsilon}$ over $H_{0}^{1}\left(\Omega ; \mathbb{R}^{3}\right)$ :

$$
J^{\epsilon}\left(\mathbf{u}^{\epsilon}\right)=\min _{\mathbf{v} \in H_{0}^{1}\left(\Omega ; \mathbb{R}^{3}\right)} J^{\epsilon}(\mathbf{v}) \quad \text { with } \quad J^{\epsilon}(\mathbf{v})=\int_{\Omega}\left(\frac{1}{2} A^{\epsilon} \varepsilon_{x}(\mathbf{v}) \cdot \varepsilon_{x}(\mathbf{v})-\mathbf{f} \cdot \mathbf{v}\right) \mathrm{d} x .
$$

As usual for the asymptotic homogenization approaches, the micro-cell $Y^{\epsilon}$ is re-scaled by introducing the "unit" cell $Y$ so that $Y^{\epsilon}=\epsilon Y$ and we assume that $|Y|=1$. In the quasi-periodic case, the properties of a point in the cell depends not only on its position but also the position of the cell in the medium. Specifically, the local elasticity tensor $A_{i j k l}^{\epsilon}$ in the unit cell can be represented by a function $\mathrm{A}_{i j k l}(\mathbf{x}, \mathbf{y})$ which is assumed to be smooth in the first variable $\mathbf{x} \in \Omega$ (Fig. 1) and $Y$-periodic in the second one $\mathbf{y} \in Y$

$$
\mathrm{A}_{i j k l}^{\epsilon}(\mathbf{x})=\mathrm{A}_{i j k l}\left(\mathbf{x}, \frac{\mathbf{x}}{\epsilon}\right) .
$$

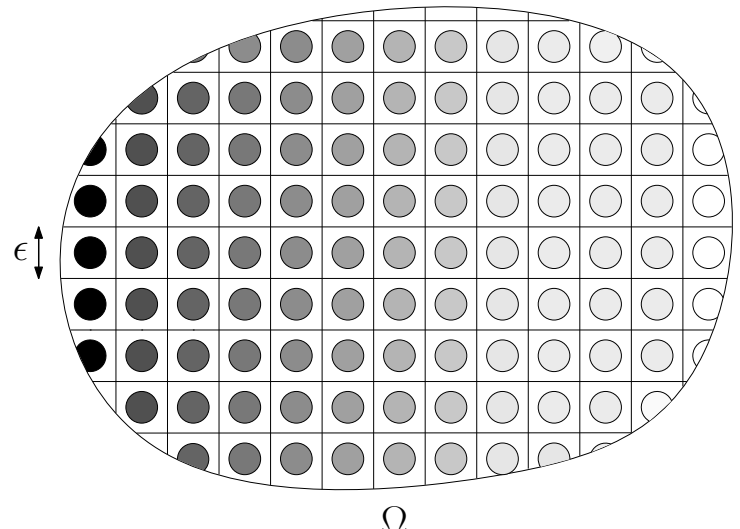

Fig. 1. A typical quasi-periodic medium considered in the paper, where the inclusions have all the same size but a stiffness which varies smoothly with their location $\mathbf{x}$ in the domain $\Omega$

Remark 1. This type of quasi-periodic medium is a particular case of a more general class of heterogeneous media where the microstructure varies smoothly. Indeed, here we assume that the cell $Y$ is always the same, independent of $x$. A more general case would consist in considering that $Y$ depends on $x$. For instance, one could consider that the dependence of the stiffness on the position reads as

$$
\mathrm{A}^{\epsilon}(x)=\mathrm{A}\left(x, \frac{\varphi(x)}{\epsilon}\right)
$$

where $\boldsymbol{\varphi}$ is a smooth diffeomorphism. In such a case, assuming that $\mathrm{A}$ is still periodic with respect to the second variable with period $Y$, the image of $\epsilon Y$ by $\varphi^{-1}$ will depend on $x$. 
By using the traditional multi-scale method like in [1,15], the displacement field $\mathbf{u}^{\epsilon}$ is represented through an asymptotic expansion in terms of the small parameter $\epsilon$

$$
\mathbf{u}^{\epsilon}(\mathbf{x})=\mathbf{u}^{0}\left(\mathbf{x}, \frac{\mathbf{x}}{\epsilon}\right)+\epsilon \mathbf{u}^{1}\left(\mathbf{x}, \frac{\mathbf{x}}{\epsilon}\right)+\epsilon^{2} \mathbf{u}^{2}\left(\mathbf{x}, \frac{\mathbf{x}}{\epsilon}\right)+\epsilon^{3} \mathbf{u}^{3}\left(\mathbf{x}, \frac{\mathbf{x}}{\epsilon}\right)+\ldots
$$

where each term $\mathbf{u}^{i}(\mathbf{x}, \mathbf{y})$ is a function of the two variables $x$ and $y$, periodic with respect to the "fast" variable $\mathbf{y}=\left(y_{1}, y_{2}, y_{3}\right)=\mathbf{x} / \epsilon$ with period $Y$. Accordingly, the stress field $\sigma^{\epsilon}$ admits the same type of expansion

$$
\sigma^{\epsilon}(\mathbf{x})=\sigma^{0}\left(\mathbf{x}, \frac{\mathbf{x}}{\epsilon}\right)+\epsilon \sigma^{1}\left(\mathbf{x}, \frac{\mathbf{x}}{\epsilon}\right)+\epsilon^{2} \sigma^{2}\left(\mathbf{x}, \frac{\mathbf{x}}{\epsilon}\right)+\epsilon^{3} \sigma^{3}\left(\mathbf{x}, \frac{\mathbf{x}}{\epsilon}\right)+\ldots
$$

Remark 2. From a regularity point of view, the solution $\mathbf{u}^{\epsilon}$ must be of finite energy and hence must belong to $H_{0}^{1}\left(\Omega ; \mathbb{R}^{3}\right)$. Therefore, one could expect that each term $\mathbf{u}^{i}$ of the expansion has the same regularity and hence that $\mathbf{u}^{i} \in H^{1}\left(\Omega \times Y ; \mathbb{R}^{3}\right)$. Moreover, the $\mathbf{u}^{i \prime}$ s must be periodic on $Y$ and one requires that they vanish on $\partial \Omega \times Y$. That leads to introduce the following set $\mathcal{U}$ at which each $\mathbf{u}^{i}$ a priori should belong

$$
\mathcal{U}=\left\{\mathbf{v} \in H^{1}\left(\Omega \times Y ; \mathbb{R}^{3}\right): \mathbf{v}=\mathbf{0} \text { on } \partial \Omega \times Y, \mathbf{v} \text { is } Y \text {-periodic }\right\} .
$$

However, we will see during the asymptotic procedure that, except $\mathbf{u}^{0}$, the subsequent $\mathbf{u}^{i}$ 's are in general less regular than expected and that they can satisfy the boundary condition on $\partial \Omega$ only under particular conditions on the microstructure. We will make some comments concerning this lack of regularity, but the study of the boundary layer effects due to the loss of the boundary conditions is outside the scope of the present paper, see Dumontet [4], Devries et al. [16] where boundary layer correctors are calculated in the periodic case.

Inserting the expansion (7) into the energy functional $J^{\epsilon}\left(\mathbf{u}^{\epsilon}\right)$, and taking into account the following derivation et integration rules

$$
\partial_{x_{l}} \rightarrow \partial_{x_{l}}+\frac{1}{\epsilon} \partial_{y_{l}}, \quad \int_{\Omega}(\cdot) \mathrm{d} \mathbf{x} \rightarrow \frac{1}{|Y|} \int_{\Omega \times Y}(\cdot) \mathrm{d} \mathbf{x} \mathrm{d} \mathbf{y}
$$

Since $|Y|=1$, the factor $1 /|Y|$ in front of the integral may be omitted. The energy functional $J^{\epsilon}\left(\mathbf{u}^{\epsilon}\right)$ becomes a series in $\epsilon$

$$
J^{\epsilon}\left(\mathbf{u}^{\epsilon}\right)=\bar{J}_{*}^{\epsilon}\left(\mathbf{u}^{0}, \mathbf{u}^{1}, \mathbf{u}^{2}, \ldots\right):=\frac{1}{\epsilon^{2}} J_{*}^{(-2)}+\frac{1}{\epsilon} J_{*}^{(-1)}+\epsilon_{*}^{(0)}+\epsilon J_{*}^{(1)}+\epsilon^{2} J_{*}^{(2)}+\ldots
$$

where

$$
J_{*}^{(p)}=J^{(p)}\left(\mathbf{u}^{0}, \ldots, \mathbf{u}^{p+1}\right),
$$

the functionals $J^{(p)}$ being defined for $p=-2$ by

$$
J^{(-2)}\left(\mathbf{v}^{0}\right)=\int_{\Omega \times Y} \frac{1}{2} A \varepsilon_{\mathbf{y}}\left(\mathbf{v}^{0}\right) \cdot \varepsilon_{\mathbf{y}}\left(\mathbf{v}^{0}\right) \mathrm{d} \mathbf{x d} \mathbf{y},
$$


and for $p \geq-1$ by

$$
\begin{aligned}
J^{(p)}\left(\mathbf{v}^{0}, \ldots, \mathbf{v}^{p+1}\right) & :=\frac{1}{2} \sum_{m+n=p} \int_{\Omega \times Y} \mathrm{~A}\left[\varepsilon_{\mathbf{x}}\left(\mathbf{v}^{m}\right)+\varepsilon_{\mathbf{y}}\left(\mathbf{v}^{m+1}\right)\right] \cdot\left[\varepsilon_{\mathbf{x}}\left(\mathbf{v}^{n}\right)+\varepsilon_{\mathbf{y}}\left(\mathbf{v}^{n+1}\right)\right] \mathrm{d} \mathbf{x} \mathrm{d} \mathbf{y} \\
& -\int_{\Omega \times Y} \mathbf{f} \cdot \mathbf{v}^{p} \mathrm{~d} \mathbf{x} \mathrm{d} \mathbf{y} .
\end{aligned}
$$

Accordingly, the minimization problem (5) leads to the following

Sequence of ordered minimization problems. Find, for $i \in \mathbb{N}$, the functions $\mathbf{u}^{i} \in \mathcal{U}$, which minimize the energy functional

$$
\bar{J}^{\epsilon}\left(\mathbf{v}^{0}, \mathbf{v}^{1}, \ldots\right)=\sum_{p \geq-2} \epsilon^{p} J^{(p)}\left(\mathbf{v}^{0}, \ldots, \mathbf{v}^{p+1}\right),
$$

over all $\mathbf{v}^{i} \in \mathcal{U}$.

In other words the formal asymptotic procedure leads to replace (5) by a sequence of minimization problems which are detailed in the next section.

Let us note that the different terms of the stress expansion are related to those of the displacement expansion by the following chain rule

$$
\sigma^{i}=\mathrm{A}\left(\varepsilon_{\mathbf{x}}\left(\mathbf{u}^{i}\right)+\varepsilon_{\mathbf{y}}\left(\mathbf{u}^{i+1}\right)\right), \quad \forall i \geq 0 .
$$

\section{SOLVING OF THE SEQUENCE OF ORDERED MINIMIZATION PROBLEMS}

\section{1. $J^{(-2)}$ and $J^{(-1)}$ minimization problems}

The field $\mathbf{u}^{0}$ has to minimize $J^{(-2)}\left(\mathbf{v}^{0}\right)$ over all $\mathbf{v}^{0} \in \mathcal{U}$. Since the stiffness tensor $\mathrm{A}$ is definite positive, we have $J^{(-2)}\left(\mathbf{v}^{0}\right) \geq 0$ with the equality only if $\varepsilon_{\mathbf{y}}\left(\mathbf{v}^{0}\right)=0$ and hence only if $\mathbf{v}^{0}$ is independent of $\mathbf{y}$. Therefore, the minimization of $J^{(-2)}$ requires that $\mathbf{u}^{0}$ is function of the macroscopic variable $\mathbf{x}$ only

$$
\mathbf{u}^{0}=\mathbf{u}^{0}(\mathbf{x})
$$

and hence $\mathbf{u}^{0}$ must belong to $H_{0}^{1}\left(\Omega ; \mathbb{R}^{3}\right)$. In turn, considering $\mathbf{v}^{0}$ such that $\mathbf{v}^{0}=\mathbf{v}^{0}(\mathbf{x})$ leads to $J^{(-1)}\left(\mathbf{v}^{0}, \mathbf{v}^{1}\right)=0$, regardless of $\mathbf{v}^{1}$ Therefore, for such $\mathbf{v}^{0}$, the energy starts at the order 0

$$
\bar{J}^{\epsilon}\left(\mathbf{v}^{0}, \mathbf{v}^{1}, \ldots\right)=J^{(0)}\left(\mathbf{v}^{0}, \mathbf{v}^{1}\right)+\epsilon J^{(1)}\left(\mathbf{v}^{0}, \mathbf{v}^{1}, \mathbf{v}^{2}\right)+\ldots
$$

\section{2. $J^{(0)}$ minimization problem}

Considering $\mathbf{v}^{0}$ independent of $\mathbf{y}$, the energy $J^{(0)}\left(\mathbf{v}^{0}, \mathbf{v}^{1}\right)$ reads as

$$
J^{(0)}\left(\mathbf{v}^{0}, \mathbf{v}^{1}\right)=\int_{\Omega \times Y} \frac{1}{2} \mathrm{~A}\left[\varepsilon_{\mathbf{x}}\left(\mathbf{v}^{0}\right)+\varepsilon_{\mathbf{y}}\left(\mathbf{v}^{1}\right)\right] \cdot\left[\varepsilon_{\mathbf{y}}\left(\mathbf{v}^{0}\right)+\varepsilon_{\mathbf{y}}\left(\mathbf{v}^{1}\right)\right] \mathrm{d} \mathbf{x} \mathrm{d} \mathbf{y}-\int_{\Omega \times Y} \mathbf{f} \cdot \mathbf{v}^{0} \mathrm{~d} \mathbf{x} \mathrm{d} \mathbf{y} .
$$

We have to find $\mathbf{u}^{0} \in H_{0}^{1}\left(\Omega ; \mathbb{R}^{3}\right)$ and $\mathbf{u}^{1} \in \mathcal{U}$ which minimize $J^{(0)}$. We proceed in two steps:

(1) First, for a given $\mathbf{v}^{0} \in H_{0}^{1}\left(\Omega ; \mathbb{R}^{3}\right)$, we minimize $J^{(0)}\left(\mathbf{v}^{0}, \cdot\right)$ over $\mathcal{U}$. 
(2) The so-obtained minimum becomes a functional of $\mathbf{v}^{0}$ that we minimize to obtain $\mathbf{u}^{0}$.

3.2.1. Minimizing with respect to $\mathbf{v}^{1}$ at given $\mathbf{v}^{0}$

At given $\mathbf{v}^{0}$, since $J^{(0)}\left(\mathbf{v}^{0}, \mathbf{v}^{1}\right)$ is a convex functional of $\mathbf{v}^{1}$, its minimizer, denoted by $\overline{\mathbf{v}}^{1}$ and which depends on $\mathbf{v}^{0}$, is such that the first derivative of $J^{(0)}\left(\mathbf{v}^{0}, \cdot\right)$ vanishes. That leads to the following variational equation for $\overline{\mathbf{v}}^{1}$

$$
\int_{\Omega \times Y} \mathrm{~A}\left[\varepsilon_{x}\left(\mathbf{v}^{0}\right)+\varepsilon_{\mathbf{y}}\left(\overline{\mathbf{v}}^{1}\right)\right] \cdot \varepsilon_{\mathbf{y}}(\mathbf{v}) \mathrm{d} \mathbf{x d} \mathbf{y}=0,
$$

which must hold for any $\mathbf{v} \in \mathcal{U}$. Let us choose $\mathbf{v}$ under the form

$$
\mathbf{v}(\mathbf{x}, \mathbf{y})=w(\mathbf{x}) \boldsymbol{\phi}(\mathbf{y}) \quad \text { with } \quad w \in \mathcal{D}(\Omega) \text { and } \boldsymbol{\phi} \in H_{\#}^{1}\left(Y ; \mathbb{R}^{3}\right) .
$$

Inserting this test function in (17) and using classic argument of calculus of variation, we get (at almost all $\mathbf{x} \in \Omega$ )

$$
\int_{\{\mathbf{x}\} \times Y} \mathrm{~A}\left[\varepsilon_{\mathbf{x}}\left(\mathbf{v}^{0}\right)+\varepsilon_{\mathbf{y}}\left(\overline{\mathbf{v}}^{1}\right)\right] \cdot \varepsilon_{\mathbf{y}}(\boldsymbol{\phi}) \mathrm{d} \mathbf{y}=0, \quad \forall \boldsymbol{\phi} \in H_{\#}^{1}\left(Y ; \mathbb{R}^{3}\right) .
$$

Since (18) brings into play the derivative of $\overline{\mathbf{v}}^{1}$ with respect to $\mathbf{y}$ only, $\overline{\mathbf{v}}^{1}$ is determined at this stage up to an arbitrary function of $\mathbf{x}$. Moreover, by linearity, $\overline{\mathbf{v}}^{1}$ can be written as follows (where the convention of summation is still used):

$$
\overline{\mathbf{v}}^{1}(\mathbf{x}, \mathbf{y})=\varepsilon_{\mathbf{x} p q}\left(\mathbf{v}^{0}\right)(\mathbf{x}) \chi^{p q}(\mathbf{x}, \mathbf{y})+\mathbf{V}^{1}(\mathbf{x})
$$

In (19), $\mathbf{V}$ is the arbitrary function of $\mathbf{x}$ and the $\operatorname{six}^{1}$ vector fields $\chi^{p q}$ are solution of the family of six elementary problems posed on $Y$ and indexed by $\mathbf{x}$, each one corresponding to the response of the unit cell to a prescribed macroscopic strain tensor. Specifically, at given $\mathbf{x}$, the $Y$-periodic field $\mathbf{y} \mapsto \chi^{p q}(\mathbf{x}, \mathbf{y})$ is solution of the following variational equation

$$
\int_{\{\mathbf{x}\} \times Y} \mathrm{~A}\left[\mathbf{e}_{p} \odot \mathbf{e}_{q}+\boldsymbol{\varepsilon}_{\mathbf{y}}\left(\chi^{p q}\right)\right] \cdot \boldsymbol{\varepsilon}_{\mathbf{y}}(\boldsymbol{\phi}) \mathrm{d} \mathbf{y}=0, \quad \forall \boldsymbol{\phi} \in H_{\#}^{1}\left(Y ; \mathbb{R}^{3}\right),
$$

where $\odot$ denotes the symmetrized tensorial product and $\mathbf{e}_{i}$ stands for the $i^{t h}$ basis vector of the cartesian coordinates. Since (20) determines $\chi^{p q}$ up to a function of $\mathbf{x}$ only, the indetermination is removed by adding the condition that the average of $\chi^{p q}$ over $Y$ vanishes:

$$
\left\langle\chi^{p q}\right\rangle=\mathbf{0} \quad \text { with } \quad\langle\cdot\rangle=\frac{1}{|Y|} \int_{Y} \cdot \mathrm{d} \mathbf{y} .
$$

Accordingly, $\mathbf{V}^{1}$ corresponds to the average of $\overline{\mathbf{v}}^{1}$ over $Y$ but remains undetermined at this stage. Let us note that the fields $\chi^{p q}$ do not depend on $\mathbf{v}^{0}$ but depend on the quasi periodic repartition of the microstructure only. To compare with what happens in the case of a periodic medium, the solution of the cell problems now depends not only on the microscopic variable $\mathbf{y}$ but also on the macroscopic one $\mathbf{x}$.

\footnotetext{
${ }^{1}$ The number of vector fields to be determined is reduced to six because of the obvious symmetry $\chi^{p q}=\chi^{q p}$.
} 
3.2.2. Minimizing with respect to $\mathbf{y}^{0}$

Using (19), the energy $J^{(0)}\left(\mathbf{v}^{0}, \mathbf{V}^{1}\right)$ becomes the following functional $\bar{J}^{(0)}$ defined for $\mathbf{v}^{0} \in H_{0}^{1}\left(\Omega ; \mathbb{R}^{3}\right)$ by

$$
\bar{J}^{(0)}\left(\mathbf{v}^{0}\right)=\int_{\Omega} \frac{1}{2} \mathrm{~A}^{(0)} \boldsymbol{\varepsilon}_{\mathbf{x}}\left(\mathbf{v}^{0}\right) \cdot \boldsymbol{\varepsilon}_{\mathbf{X}}\left(\mathbf{v}^{0}\right) \mathrm{d} \mathbf{x}-\int_{\Omega} \mathbf{f} \cdot \mathbf{v}^{0} \mathrm{~d} \mathbf{x} .
$$

In (22) $A^{(0)}(\mathbf{x})$ is the (classical) homogenized stiffness tensor at $\mathbf{x}$ which is symmetric, positive definite and depends on $\mathbf{x}$ because of the quasi-periodicity assumption. Specifically, owing to (20), the components of $A^{(0)}(\mathbf{x})$ can be written under the two following forms

$$
\begin{aligned}
\mathrm{A}_{m n p q}^{(0)}(\mathbf{x}) & =\int_{\{\mathbf{x}\} \times Y} \mathrm{~A}\left[\mathbf{e}_{m} \odot \mathbf{e}_{n}+\varepsilon_{\mathbf{y}}\left(\chi^{m n}\right)\right] \cdot\left[\mathbf{e}_{p} \odot \mathbf{e}_{q}+\varepsilon_{\mathbf{y}}\left(\chi^{p q}\right)\right] \mathrm{d} \mathbf{y} \\
& =\int_{\{\mathbf{x}\} \times Y} \mathrm{~A}_{i j p q}\left[\delta_{i m} \delta_{j n}+\varepsilon_{\mathbf{y} i j}\left(\chi^{m n}\right)\right] \mathrm{d} \mathbf{y} .
\end{aligned}
$$

The symmetry and the positivity of $A^{(0)}$ appear more clearly from (23), but the form (24) will be useful later. Therefore $\bar{J}^{(0)}$ is convex and coercive on $H_{0}^{1}\left(\Omega ; \mathbb{R}^{3}\right)$. So its minimizer $\mathbf{u}^{0}$ is unique and such that

$$
\int_{\Omega}\left(\mathrm{A}^{(0)} \varepsilon_{\mathbf{x}}\left(\mathbf{u}^{0}\right) \cdot \varepsilon_{\mathbf{x}}(\mathbf{v})-\mathbf{f} \cdot \mathbf{v}\right) \mathrm{d} \mathbf{x}=0, \quad \forall \mathbf{v} \in H_{0}^{1}\left(\Omega ; \mathbb{R}^{3}\right) .
$$

Accordingly, $\mathbf{u}^{0}$ is the unique solution of the following linear elastic problem defined on the "homogenized" body (that is to say, the body whose microstructure has been removed and replaced by its effective stiffness $A^{(0)}$ )

$$
\begin{cases}\operatorname{div}_{\mathbf{x}}\left(A^{(0)} \varepsilon_{\mathbf{x}}\left(\mathbf{u}^{0}\right)\right)+\mathbf{f}=0, & \text { in } \Omega \\ \mathbf{u}^{0}=0, & \text { on } \partial \Omega\end{cases}
$$

Once this problem is solved, $\mathbf{u}^{0}$ is known and we obtain from (19) that the second term $\mathbf{u}^{1}$ of the expansion of $\mathbf{u}^{\epsilon}$ can read as

$$
\mathbf{u}^{1}(\mathbf{x}, \mathbf{y})=\varepsilon_{\mathbf{x} p q}\left(\mathbf{u}^{0}\right)(\mathbf{x}) \chi^{p q}(\mathbf{x}, \mathbf{y})+\left\langle\mathbf{u}^{1}\right\rangle(\mathbf{x}),
$$

its average value $\left\langle\mathbf{u}^{1}\right\rangle$ only remaining unknown at this stage. Moreover, the first order stress field $\sigma^{0}$ is also known and given by

$$
\sigma^{0}(\mathbf{x}, \mathbf{y})=\mathrm{A}(\mathbf{x}, \mathbf{y})\left(\varepsilon_{\mathbf{x}}\left(\mathbf{u}^{0}\right)(\mathbf{x})+\varepsilon_{\mathbf{x} p q}\left(\mathbf{u}^{0}\right)(\mathbf{x}) \varepsilon_{\mathbf{y}}\left(\chi^{p q}\right)(\mathbf{x}, \mathbf{y})\right) .
$$

Let us note that $\sigma^{0}$ depends linearly on the macroscopic strain $\varepsilon_{\mathbf{x}}\left(\mathbf{u}^{0}\right)$,

$$
\sigma^{0}(\mathbf{x}, \mathbf{y})=\mathbf{a}^{0}(\mathbf{x}, \mathbf{y}) \varepsilon_{\mathbf{x}}\left(\mathbf{u}^{0}\right),
$$

where the fourth-order stiffness tensor field $a^{0}$ (sometimes called the stress localization field) depends only on the microstructure

$$
\mathrm{a}_{i j k l}^{0}=\mathrm{A}_{i j k l}+\mathrm{A}_{i j p q} \varepsilon_{y p q}\left(\chi^{k l}\right) .
$$


The macroscopic stress field of order 0 is the average value of $\sigma^{0}$ on $Y$ and, by virtue of (24), is associated to the strain field of order 0 by the effective relation of order 0

$$
\left\langle\sigma^{0}\right\rangle=\mathrm{A}^{(0)} \varepsilon_{\mathbf{x}}\left(\mathbf{u}^{0}\right) .
$$

Therefore $A^{(0)}$ is nothing but that the average value of $a^{0}: A^{(0)}=\left\langle a^{0}\right\rangle$.

Let us make some comments concerning the regularity and the boundary conditions satisfied by $\mathbf{u}^{1}$ in the spirit of Remark 2 .

Remark 3. (i) In order that $\mathbf{u}^{1}$ is in $H^{1}\left(\Omega ; \mathbb{R}^{3}\right)$, it is necessary that $\mathbf{u}^{0}$ is in $H^{2}\left(\Omega ; \mathbb{R}^{3}\right)$ which is the case if the effective stiffness tensor $\mathrm{A}^{(0)}$ and the body forces $\mathbf{f}$ are smooth functions of $\boldsymbol{x}$.

(ii) The verification of the boundary condition $\mathbf{u}^{1}=0$ on $\partial \Omega \times Y$ is more delicate. Indeed, in general $\varepsilon_{x}\left(\mathbf{u}^{0}\right)$ does not vanish on $\partial \Omega$. Therefore, the unique possibility for $\mathbf{u}^{1}$ to satisfy the boundary condition is that the six fields $\chi^{p q}$ vanish on $\partial \Omega \times Y$ (otherwise boundary layer effects will appear). This is the case when the body is homogeneous in a neighborhood of the boundary, i.e. when $A$ does not depend on $\mathbf{y}$ when $\boldsymbol{x}$ is close to $\partial \Omega$. In such a case it suffices that $\left\langle\mathbf{u}^{1}\right\rangle=0$ on $\partial \Omega$ in order that the boundary condition is satisfied.

In the sequel, we will assume that all the conditions are satisfied in order that $\mathbf{u}^{1}$ actually belongs to $H_{0}^{1}\left(\Omega ; \mathbb{R}^{3}\right)$.

\subsection{Calculation of $J^{(1)}\left(\mathbf{u}^{0}, \mathbf{u}^{1}, \mathbf{u}^{2}\right)$}

By the definition (13) one gets

$$
J^{(1)}\left(\mathbf{u}^{0}, \mathbf{u}^{1}, \mathbf{u}^{2}\right)=\int_{\Omega \times Y}\left(\mathrm{~A}\left[\varepsilon_{\mathbf{x}}\left(\mathbf{u}^{0}\right)+\varepsilon_{\mathbf{y}}\left(\mathbf{u}^{1}\right)\right] \cdot\left[\varepsilon_{\mathbf{x}}\left(\mathbf{u}^{1}\right)+\varepsilon_{\mathbf{y}}\left(\mathbf{u}^{2}\right)\right]-\mathbf{f} \cdot \mathbf{u}^{1}\right) \mathrm{d} \mathbf{x d} \mathbf{y} .
$$

Using (18) with $\left(\mathbf{u}^{0}, \mathbf{u}^{1}, \mathbf{u}^{2}\right)$ in place of $\left(\mathbf{v}^{0}, \overline{\mathbf{v}}^{1}, \mathbf{v}\right)$ one obtains

$$
\int_{\Omega \times Y} \mathrm{~A}\left[\varepsilon_{\mathbf{x}}\left(\mathbf{u}^{0}\right)+\varepsilon_{\mathbf{y}}\left(\mathbf{u}^{1}\right)\right] \cdot \varepsilon_{\mathbf{y}}\left(\mathbf{u}^{2}\right) \mathrm{d} \mathbf{x d} \mathbf{y}=0 .
$$

Using (25) with $\mathbf{v}=\left\langle\mathbf{u}^{1}\right\rangle$ gives

$$
\int_{\Omega}\left(A^{(0)} \varepsilon_{\mathbf{x}}\left(\mathbf{u}^{0}\right) \cdot \varepsilon_{\mathbf{x}}\left(\left\langle\mathbf{u}^{1}\right\rangle\right)-\mathbf{f} \cdot\left\langle\mathbf{u}^{1}\right\rangle\right) \mathrm{d} \mathbf{x}=0 .
$$

Therefore, using the form (24) for $\mathrm{A}^{(0)}$, the previous equality reads also as

$$
\int_{\Omega \times Y}\left(\mathrm{~A}\left[\varepsilon_{\mathbf{x}}\left(\mathbf{u}^{0}\right)+\varepsilon_{\mathbf{y}}\left(\mathbf{u}^{1}\right)\right] \cdot \varepsilon_{\mathbf{x}}\left(\left\langle\mathbf{u}^{1}\right\rangle\right)-\mathbf{f} \cdot\left\langle\mathbf{u}^{1}\right\rangle\right) \mathrm{d} \mathbf{x} \mathrm{d} \mathbf{y}=0 .
$$

Inserting (32) and (33) into (31) shows that $J^{(1)}\left(\mathbf{u}^{0}, \mathbf{u}^{1}, \mathbf{u}^{2}\right)$ depends in fact only on $\mathbf{u}^{0}$, but not on $\left\langle\mathbf{u}^{1}\right\rangle$ and $\mathbf{u}^{2}$. Therefore, $J_{*}^{(1)}:=J^{(1)}\left(\mathbf{u}^{0}, \mathbf{u}^{1}, \mathbf{u}^{2}\right)$ can be considered as known at this stage.

Furthermore, let us remark that the energy $J_{*}^{(1)}$ can be seen as a quadratic form of $\mathbf{u}^{0}$ which involves new homogenized stiffness tensors that we propose to identify. Indeed, 
since $\mathbf{f}$ does not depend on $\mathbf{y}$, the last term in (31) can read as

$$
\int_{\Omega \times Y} \mathbf{f} \cdot \mathbf{u}^{1} \mathrm{~d} \mathbf{x d} \mathbf{y}=\int_{\Omega} \mathbf{f} \cdot\left\langle\mathbf{u}^{1}\right\rangle \mathrm{d} \mathbf{x}
$$

Then, using (28), (32) and (33), (31) becomes

$$
J_{*}^{(1)}=\int_{\Omega \times Y} \sigma^{0} \cdot \varepsilon_{\mathbf{x}}\left(\varepsilon_{\mathbf{x} i j}\left(\mathbf{u}^{0}\right) \chi^{i j}\right) \mathrm{d} \mathbf{x d} \mathbf{y} .
$$

For a future use, it is more convenient to make an integration by parts of the integrand to obtain

$$
J_{*}^{(1)}=-\int_{\Omega \times Y} \operatorname{div}_{\mathbf{x}} \sigma^{0} \cdot \chi^{i j} \varepsilon_{\mathbf{x} i j}\left(\mathbf{u}^{0}\right) \mathrm{d} \mathbf{x d} \mathbf{y},
$$

the boundary term on $\partial \Omega \times Y$ cancelling by virtue of Remark 3 . Using (29) and expanding the derivative with respect to $x$ lead to

$$
\operatorname{div}_{\mathbf{x}} \sigma^{0}=\partial_{x_{q}}\left(a_{p q k l}^{0}\right) \varepsilon_{\mathbf{x} k l}\left(\mathbf{u}^{0}\right) \mathbf{e}_{p}+a_{p q k l}^{0} g_{x k l q}\left(\mathbf{u}^{0}\right) \mathbf{e}_{p},
$$

where $\mathbf{g}_{\mathbf{x}}(\mathbf{u})$ denotes the gradient of the symmetrized gradient of $\mathbf{u}$ (all the derivatives are with respect to $\mathbf{x}$ ) whose components are given by

$$
g_{\mathbf{x} i j k}(\mathbf{u})=\partial_{x_{k}}\left(\varepsilon_{\mathbf{x} i j}(\mathbf{u})\right)=\frac{1}{2}\left(\frac{\partial^{2} u_{i}}{\partial x_{j} \partial x_{k}}+\frac{\partial^{2} u_{j}}{\partial x_{i} \partial x_{k}}\right) .
$$

Inserting (34) into $J_{*}^{(1)}$ leads to

$$
J_{*}^{(1)}=\int_{\Omega}\left(\mathrm{A}^{(1)} \varepsilon_{\mathbf{x}}\left(\mathbf{u}^{0}\right) \cdot \varepsilon_{\mathbf{x}}\left(\mathbf{u}^{0}\right) \mathrm{d} \mathbf{x}+\mathrm{B}^{(0)} \mathbf{g}_{\mathbf{x}}\left(\mathbf{u}^{0}\right) \cdot \varepsilon_{\mathbf{x}}\left(\mathbf{u}^{0}\right)\right) \mathrm{d} \mathbf{x},
$$

where appear the fourth-order tensor field $A^{(1)}$ and the fifth-order tensor field $B^{(0)}$ defined by

$$
\mathrm{A}_{i j k l}^{(1)}(\mathbf{x})=-\int_{\{\mathbf{x}\} \times Y} \partial_{x_{q}}\left(a_{p q k l}^{0}\right) \chi_{p}^{i j} \mathrm{~d} \mathbf{y}, \quad \mathrm{B}_{i j k l q}^{(0)}(\mathbf{x})=-\int_{\{\mathbf{x}\} \times Y} a_{p q k l}^{0} \chi_{p}^{i j} \mathrm{~d} \mathbf{y} .
$$

These two tensor fields depend only on the microstructure and can be considered as two other effective stiffness tensor fields. Let us note also that $A^{(1)}$ has the minor symmetries, not the major one (but its symmetric part only is involved in (36))

$$
\mathrm{A}_{i j k l}^{(1)}=\mathrm{A}_{j i k l}^{(1)}=\mathrm{A}_{i j l k^{\prime}}^{(1)} \quad \mathrm{A}_{i j k l}^{(1)} \neq \mathrm{A}_{k l i j^{\prime}}^{(1)}
$$

and that $\mathrm{B}^{(1)}$ has the following symmetries

$$
\mathrm{B}_{i j k l m}^{(0)}=\mathrm{B}_{j i k l m}^{(0)}=\mathrm{B}_{i j k m}^{(0)} .
$$

It remains to determine $\left\langle\mathbf{u}^{1}\right\rangle$. It will be given by minimizing $J^{(2)}$. 


\section{4. $J^{(2)}$ minimization problem}

Let us consider $J^{(2)}\left(\mathbf{u}^{0}, \mathbf{v}^{1}, \mathbf{v}^{2}, \mathbf{v}^{3}\right)$ with

$$
\mathbf{v}^{1}=\varepsilon_{\mathbf{x} p q}\left(\mathbf{u}^{0}\right) \chi^{p q}+\left\langle\mathbf{v}^{1}\right\rangle, \quad\left\langle\mathbf{v}^{1}\right\rangle \in H_{0}^{1}\left(\Omega ; \mathbb{R}^{3}\right)
$$

and $\mathbf{v}^{2}, \mathbf{v}^{3}$ in $\mathcal{U}$. Note that $\mathbf{y}^{1}$ differs from $\mathbf{u}^{1}$ only by its average value and hence that $\varepsilon_{\mathbf{y}}\left(\mathbf{v}^{1}\right)=\varepsilon_{\mathbf{y}}\left(\mathbf{u}^{1}\right)$. By virtue of (13), one gets

$$
\begin{array}{r}
J^{(2)}\left(\mathbf{u}^{0}, \mathbf{v}^{1}, \mathbf{v}^{2}, \mathbf{v}^{3}\right)=\int_{\Omega \times Y} \frac{1}{2} \mathbf{A}\left[\varepsilon_{\mathbf{x}}\left(\mathbf{v}^{1}\right)+\varepsilon_{\mathbf{y}}\left(\mathbf{v}^{2}\right)\right] \cdot\left[\varepsilon_{\mathbf{x}}\left(\mathbf{v}^{1}\right)+\varepsilon_{\mathbf{y}}\left(\mathbf{v}^{2}\right)\right] \mathrm{d} \mathbf{x d} \mathbf{y}+ \\
\quad+\int_{\Omega \times Y} A\left[\varepsilon_{\mathbf{x}}\left(\mathbf{u}^{0}\right)+\varepsilon_{\mathbf{y}}\left(\mathbf{u}^{1}\right)\right] \cdot\left[\varepsilon_{\mathbf{x}}\left(\mathbf{v}^{2}\right)+\varepsilon_{\mathbf{y}}\left(\mathbf{v}^{3}\right)\right] \mathrm{d} \mathbf{x} \mathrm{d} \mathbf{y}-\int_{\Omega \times Y} \mathbf{f} \cdot \mathbf{v}^{2} \mathrm{~d} \mathbf{x} \mathrm{d} \mathbf{y}
\end{array}
$$

Using (18) with $\left(\mathbf{u}^{0}, \mathbf{u}^{1}, \mathbf{v}^{3}\right)$ in place of $\left(\mathbf{v}^{0}, \mathbf{v}^{1}, \mathbf{v}\right)$ one obtains

$$
\int_{\Omega \times Y} \mathrm{~A}\left[\varepsilon_{\mathbf{x}}\left(\mathbf{u}^{0}\right)+\varepsilon_{\mathbf{y}}\left(\mathbf{u}^{1}\right)\right] \cdot \varepsilon_{\mathbf{y}}\left(\mathbf{v}^{3}\right) \mathrm{d} \mathbf{x d} \mathbf{y}=0,
$$

and hence $J^{(2)}\left(\mathbf{u}^{0}, \mathbf{v}^{1}, \mathbf{v}^{2}, \mathbf{v}^{3}\right)$ does not depend on the choice of $\mathbf{v}^{3}$ in $\mathcal{U}$. By virtue of (25), see also (33), one has

$$
\int_{\Omega \times Y}\left(\mathrm{~A}\left[\varepsilon_{\mathbf{x}}\left(\mathbf{u}^{0}\right)+\varepsilon_{\mathbf{y}}\left(\mathbf{u}^{1}\right)\right] \cdot \varepsilon_{\mathbf{x}}\left(\left\langle\mathbf{v}^{2}\right\rangle\right)-\mathbf{f} \cdot\left\langle\mathbf{v}^{2}\right\rangle\right) \mathrm{d} \mathbf{x} \mathrm{d} \mathbf{y}=0,
$$

and hence, since $\mathbf{u}^{0}$ is known and since $\varepsilon_{\mathbf{y}}\left(\left\langle\mathbf{v}^{2}\right\rangle\right)=0, J^{(2)}\left(\mathbf{u}^{0}, \mathbf{v}^{1}, \mathbf{v}^{2}, \mathbf{v}^{3}\right)$ only depends on $\left\langle\mathbf{v}^{1}\right\rangle$ and $\mathbf{v}^{2}-\left\langle\mathbf{v}^{2}\right\rangle$. Therefore, the problem consists in minimizing, for $\mathbf{u}^{0}$ given in $H_{0}^{1}\left(\Omega ; \mathbb{R}^{3}\right)$, the following quadratic functional $\bar{J}^{(2)}$ defined on $H_{0}^{1}\left(\Omega ; \mathbb{R}^{3}\right) \times \mathcal{U}_{0}$

$$
\begin{aligned}
& \bar{J}^{(2)}(\langle\mathbf{v}\rangle, \mathbf{w})=\int_{\Omega \times Y} \mathrm{~A}\left[\varepsilon_{\mathbf{x}}\left(\mathbf{u}^{0}\right)+\varepsilon_{\mathbf{x} p q}\left(\mathbf{u}^{0}\right) \varepsilon_{\mathbf{y}}\left(\chi^{p q}\right)\right] \cdot \varepsilon_{\mathbf{x}}(\mathbf{w}) \mathrm{d} \mathbf{x} \mathrm{d} \mathbf{y}+ \\
& +\int_{\Omega \times Y} \frac{1}{2} \mathrm{~A}\left[\varepsilon_{\mathbf{x}}(\langle\mathbf{v}\rangle)+\varepsilon_{\mathbf{y}}(\mathbf{w})+\varepsilon_{\mathbf{x}}\left(\varepsilon_{\mathbf{x} p q}\left(\mathbf{u}^{0}\right) \chi^{p q}\right)\right] \cdot\left[\varepsilon_{\mathbf{x}}(\langle\mathbf{v}\rangle)+\varepsilon_{\mathbf{y}}(\mathbf{w})+\varepsilon_{\mathbf{x}}\left(\varepsilon_{\mathbf{x} p q}\left(\mathbf{u}^{0}\right) \chi^{p q}\right)\right] \mathrm{d} \mathbf{x} \mathrm{d} \mathbf{y},
\end{aligned}
$$

where $\mathcal{U}_{0}$ denotes the linear subset of $\mathcal{U}$ made of fields defined on $\Omega \times Y$ whose average value with respect to $Y$ vanishes (almost) everywhere in $\Omega$

$$
U_{0}=\{\mathbf{v} \in \mathcal{U}:\langle\mathbf{v}\rangle(\mathbf{x})=\mathbf{0}, \forall \mathbf{x} \in \Omega\} .
$$

The minimizer of $\bar{J}^{(2)}$ is $\left(\left\langle\mathbf{u}^{1}\right\rangle, \mathbf{u}^{2}-\left\langle\mathbf{u}^{2}\right\rangle\right)$ (which means that $\mathbf{u}^{2}$ will be determined up to a function of $\mathbf{x}$ at this stage). To minimize $\bar{J}^{(2)}$, we proceed in three steps:

(1) First, for a given $\langle\mathbf{v}\rangle \in H_{0}^{1}\left(\Omega ; \mathbb{R}^{3}\right)$, we minimize $\bar{J}^{(2)}(\langle\mathbf{v}\rangle, \cdot)$ over $\mathcal{U}_{0}$.

(2) The so-obtained minimum becomes a functional of $\langle\mathbf{v}\rangle$ defined on $H_{0}^{1}\left(\Omega ; \mathbb{R}^{3}\right)$ which involves six effective stiffness tensor fields.

(3) Finally, minimizing that functional gives $\left\langle\mathbf{u}^{1}\right\rangle$ and hence $\mathbf{u}^{2}-\left\langle\mathbf{u}^{2}\right\rangle$. 


\subsubsection{Minimizing with respect to $\mathbf{w}$ at given $\langle\mathbf{v}\rangle$}

At given $\langle\mathbf{v}\rangle$, since $\bar{J}^{(2)}(\langle\mathbf{v}\rangle, \mathbf{w})$ is a convex functional of $\mathbf{w}$, its minimizer, denoted by $\mathbf{W}$ and which depends on $\mathbf{u}^{0}$ and $\langle\mathbf{v}\rangle$, is such that the first derivative of $\bar{J}^{(2)}(\langle\mathbf{v}\rangle, \cdot)$ vanishes. Using the relation (28) for $\sigma^{0}$, that leads to the following variational equation for $\overline{\mathbf{w}}$

$$
0=\int_{\Omega \times Y}\left(\mathrm{~A}\left[\varepsilon_{\mathbf{y}}(\overline{\mathbf{w}})+\varepsilon_{\mathbf{x}}(\langle\mathbf{v}\rangle)+\varepsilon_{\mathbf{x}}\left(\varepsilon_{\mathbf{x} p q}\left(\mathbf{u}^{0}\right) \chi^{p q}\right)\right] \cdot \varepsilon_{\mathbf{y}}(\mathbf{w})+\sigma^{0} \cdot \varepsilon_{\mathbf{x}}(\mathbf{w})\right) \mathrm{d} \mathbf{x} \mathrm{d} \mathbf{y},
$$

which must hold for any $\mathbf{w} \in \mathcal{U}_{0}$. Because of the presence of the gradient of $\mathbf{w}$ with respect to $\mathbf{x}$ in the last term of (42) we cannot directly use classical arguments to suppress the integral over $\Omega$ for obtaining local problems posed at almost all points $\mathbf{x}$ of $\Omega$. We must before make an integration by parts of that term. So, integrating by parts the last term of (42) gives

$$
0=\int_{\Omega \times Y}\left(\mathrm{~A}\left[\varepsilon_{\mathbf{y}}(\overline{\mathbf{w}})+\varepsilon_{\mathbf{x}}(\langle\mathbf{v}\rangle)+\varepsilon_{\mathbf{x}}\left(\varepsilon_{\mathbf{x} p q}\left(\mathbf{u}^{0}\right) \chi^{p q}\right)\right] \cdot \varepsilon_{\mathbf{y}}(\mathbf{w})-\operatorname{div}_{\mathbf{x}} \sigma^{0} \cdot \mathbf{w}\right) \mathrm{d} \mathbf{x d} \mathbf{y},
$$

the boundary term on $\partial \Omega$ cancelling because $\mathbf{w} \in \mathcal{U}$. Let us choose $\mathbf{w}$ under the form

$$
\mathbf{w}(\mathbf{x}, \mathbf{y})=\varphi(\mathbf{x}) \boldsymbol{\phi}(\mathbf{y}) \text { with } \varphi \in \mathcal{D}(\Omega) \text { and } \boldsymbol{\phi} \in H_{\# 0}^{1}\left(Y ; \mathbb{R}^{3}\right),
$$

where $H_{\# 0}^{1}\left(Y ; \mathbb{R}^{3}\right)$ denotes the $Y$-periodic fields with zero average value

$$
H_{\# 0}^{1}\left(Y ; \mathbb{R}^{3}\right)=\left\{\boldsymbol{\phi} \in H_{\#}^{1}\left(Y ; \mathbb{R}^{3}\right):\langle\boldsymbol{\phi}\rangle=0\right\} .
$$

Inserting this test function into (43), we can now use classical argument of calculus of variation to get (at almost all $\mathbf{x} \in \Omega$ ):

$$
\int_{\{\mathbf{x}\} \times Y} \mathrm{~A}\left[\varepsilon_{\mathbf{y}}(\overline{\mathbf{w}})+\varepsilon_{\mathbf{x}}(\langle\mathbf{v}\rangle)+\varepsilon_{\mathbf{x}}\left(\varepsilon_{\mathbf{x} p q}\left(\mathbf{u}^{0}\right) \chi^{p q}\right)\right] \cdot \varepsilon_{\mathbf{y}}(\phi) d \mathbf{y}=\int_{\{\mathbf{x}\} \times Y} \operatorname{div}_{\mathbf{x}} \sigma^{0} \cdot \boldsymbol{\phi} \mathrm{d} \mathbf{y},
$$

which must hold for any $\phi \in H_{\# 0}^{1}\left(Y ; \mathbb{R}^{3}\right)$.

Let us note that both $\varepsilon_{\mathbf{x}}\left(\varepsilon_{\mathbf{x} p q}\left(\mathbf{u}^{0}\right) \chi^{p q}\right)$ and $\operatorname{div}_{\mathbf{x}} \sigma^{0}$ depend linearly on $\varepsilon_{\mathbf{x}}(\mathbf{u})$ and its gradient $\mathbf{g}_{\mathbf{x}}\left(\mathbf{u}^{0}\right)$. The dependency of the latter is given by (34) whereas for the former one gets

$$
\varepsilon_{\mathbf{x} i j}\left(\varepsilon_{\mathbf{x} k l}\left(\mathbf{u}^{0}\right) \chi^{k l}\right)=\varepsilon_{\mathbf{x} k l}\left(\mathbf{u}^{0}\right) \varepsilon_{\mathbf{x} i j}\left(\chi^{k l}\right)+\frac{1}{2}\left(g_{\mathbf{x} k l i}\left(\mathbf{u}^{0}\right) \chi_{j}^{k l}+g_{\mathbf{x} k l j}\left(\mathbf{u}^{0}\right) \chi_{i}^{k l}\right) .
$$

Using these properties of linearity allow us to decompose $\overline{\mathbf{w}}$ as the combination of fields depending on the microstructure only. Specifically, $\overline{\mathbf{w}}$ depends linearly on the six (independent) components of $\varepsilon_{\mathbf{x}}(\langle\mathbf{v}\rangle)$, the six (independent) components of $\varepsilon_{\mathbf{x}}\left(\mathbf{u}^{0}\right)$ and the eighteen (independent) components of $\mathbf{g}_{\mathbf{x}}\left(\mathbf{u}^{0}\right)$, and hence can be read as

$$
\overline{\mathbf{w}}(\mathbf{x}, \mathbf{y})=\varepsilon_{\mathbf{x} p q}(\langle\mathbf{v}\rangle)(\mathbf{x}) \chi^{p q}(\mathbf{x}, \mathbf{y})+\varepsilon_{\mathbf{x} p q}\left(\mathbf{u}^{0}\right)(\mathbf{x}) \boldsymbol{\psi}^{p q}(\mathbf{x}, \mathbf{y})+g_{\mathbf{x} p q r}\left(\mathbf{u}^{0}\right)(\mathbf{x}) \boldsymbol{\xi}^{p q r}(\mathbf{x}, \mathbf{y})
$$

In (47), the quasi periodic displacement fields associated with $\varepsilon_{\mathbf{x}}(\mathbf{v})$ are the same fields $\chi^{p q}$ as those defined by (20) and (21), whereas the other ones, $\psi^{p q}$ and $\xi^{p q r}$, associated 
with the strain $\varepsilon_{\mathbf{x}}\left(\mathbf{u}^{0}\right)$ and its gradient $\mathbf{g}_{\mathbf{x}}\left(\mathbf{u}^{0}\right)$ respectively, are new quasi periodic fields which are solution in $\mathcal{U}_{0}$ of the following problems defined on the unit cell $\{\mathbf{x}\} \times Y$ :

$$
\begin{gathered}
\text { Find } \boldsymbol{\psi}^{p q} \in H_{\# 0}^{1}\left(Y ; \mathbb{R}^{3}\right) \text { such that } \forall \boldsymbol{\phi} \in H_{\# 0}^{1}\left(Y ; \mathbb{R}^{3}\right), \\
\int_{\{\mathbf{x}\} \times Y} \mathrm{~A}\left[\varepsilon_{\mathbf{y}}\left(\boldsymbol{\psi}^{p q}\right)+\varepsilon_{\mathbf{x}}\left(\chi^{p q}\right)\right] \cdot \varepsilon_{\mathbf{y}}(\boldsymbol{\phi}) \mathrm{d} \mathbf{y}=\int_{\{\mathbf{x}\} \times Y} \partial_{x_{j}}\left(a_{i j p q}^{0}\right) \phi_{i} \mathrm{~d} \mathbf{y} ; \\
\text { Find } \boldsymbol{\xi}^{p q r} \in H_{\# 0}^{1}\left(Y ; \mathbb{R}^{3}\right) \text { such that } \forall \boldsymbol{\phi} \in H_{\# 0}^{1}\left(Y ; \mathbb{R}^{3}\right), \\
\int_{\{\mathbf{x}\} \times Y} \mathrm{~A}\left[\varepsilon_{\mathbf{y}}\left(\boldsymbol{\xi}^{p q r}\right)+\chi^{p q} \odot \mathbf{e}_{r}\right] \cdot \varepsilon_{\mathbf{y}}(\boldsymbol{\phi}) \mathrm{d} \mathbf{y}=\int_{\{\mathbf{x}\} \times Y} \mathbf{a}_{i r p q}^{0} \phi_{i} \mathrm{~d} \mathbf{y} .
\end{gathered}
$$

Remark 4. Concerning the regularity and the boundary conditions satisfied by $\overline{\mathbf{w}}$ :

(i) The existence of $\boldsymbol{\psi}^{p q}$ and $\boldsymbol{\xi}^{p q r}$ is not guaranteed but depends on the regularity of the fields $\chi^{p q}$ on $x$ and hence on the microstructure. This regularity must be checked on a case by case basis. On the other hand, the uniqueness is ensured once the existence is established.

(ii) Even if a solution $\psi^{p q}$ and $\boldsymbol{\xi}^{p q r}$ exists for each "elementary problem", the field $\mathbf{w}$ belongs to $H^{1}\left(\Omega \times Y ; \mathbb{R}^{3}\right)$ only if $\mathbf{u}^{0}$ and $\langle\mathbf{v}\rangle$ are smooth enough.

(iii) The verification of the boundary condition $\overline{\mathbf{w}}=0$ on $\partial \Omega \times Y$ is as delicate as for $\mathbf{u}^{1}$. Indeed, in general neither $\varepsilon_{x}(\langle\mathbf{v}\rangle)$, nor $\varepsilon_{x}\left(\mathbf{u}^{0}\right)$ and nor $\mathbf{g}_{x}\left(\mathbf{u}^{0}\right)$ vanish on $\partial \Omega$. Therefore, the unique possibility for $\overline{\mathbf{w}}$ to satisfy the boundary condition is that all the fields $\chi^{p q}, \psi^{p q}$ and $\boldsymbol{\xi}^{\text {pqr }}$ vanish on $\partial \Omega \times Y$. This is still the case when the body is homogeneous in a neighborhood of the boundary.

In the sequel, we will assume that all the conditions are satisfied in order that $\overline{\mathbf{w}}$ exists and belongs to $H_{0}^{1}\left(\Omega ; \mathbb{R}^{3}\right)$.

\subsubsection{Expression of $\overline{\bar{J}}(2)$}

Inserting (47) into (40) obtains a functional $\overline{\bar{J}}^{(2)}$ of $\langle\mathbf{v}\rangle$ defined on $H_{0}^{1}\left(\Omega, \mathbb{R}^{3}\right)$. The functional $\overline{\bar{J}}^{(2)}$ contains several new effective stiffness tensor fields. Specifically, after some tedious calculations which are not detailed here, one eventually gets

$$
\begin{gathered}
\overline{\bar{J}}^{(2)}(\langle\mathbf{v}\rangle)=\int_{\Omega}\left(\frac{1}{2} \mathrm{~A}^{(0)} \boldsymbol{\varepsilon}_{\mathbf{x}}(\langle\mathbf{v}\rangle) \cdot \varepsilon_{\mathbf{x}}(\langle\mathbf{v}\rangle)+\left(\mathrm{A}^{(1)} \boldsymbol{\varepsilon}_{\mathbf{x}}\left(\mathbf{u}^{0}\right)+\mathrm{B}^{(0)} \mathbf{g}_{\mathbf{x}}\left(\mathbf{u}^{0}\right)\right) \cdot \varepsilon_{\mathbf{x}}(\langle\mathbf{v}\rangle)\right) \mathrm{d} \mathbf{x} \\
+\int_{\Omega}\left(\frac{1}{2} \mathrm{~A}^{(2)} \boldsymbol{\varepsilon}_{\mathbf{x}}\left(\mathbf{u}^{0}\right) \cdot \boldsymbol{\varepsilon}_{\mathbf{x}}\left(\mathbf{u}^{0}\right)+\mathrm{B}^{(1)} \mathbf{g}_{\mathbf{x}}\left(\mathbf{u}^{0}\right) \cdot \boldsymbol{\varepsilon}_{\mathbf{x}}\left(\mathbf{u}^{0}\right)+\frac{1}{2} C^{(0)} \mathbf{g}_{\mathbf{x}}\left(\mathbf{u}^{0}\right) \cdot \mathbf{g}_{\mathbf{x}}\left(\mathbf{u}^{0}\right)\right) \mathrm{d} \mathbf{x},
\end{gathered}
$$

where $A^{(0)}, A^{(1)}$ and $B^{(0)}$ are the already defined stiffness tensor fields, see (23) and (37), whereas $A^{(2)}, B^{(1)}$ and $C^{(0)}$ are the new stiffness tensor fields given by

$$
\begin{aligned}
\mathrm{A}_{i j k l}^{(2)} & =\int_{Y}\left(\mathrm{~A} \varepsilon_{x}\left(\chi^{i j}\right) \cdot \varepsilon_{x}\left(\chi^{k l}\right)-\mathrm{A} \varepsilon_{y}\left(\psi^{i j}\right) \cdot \varepsilon_{y}\left(\psi^{k l}\right)\right) \mathrm{d} \mathbf{y}, \\
\mathrm{B}_{i j k l m}^{(1)} & =\int_{Y}\left(\mathrm{~A} \varepsilon_{\mathbf{x}}\left(\chi^{i j}\right) \cdot\left(\chi^{k l} \odot \mathbf{e}_{m}\right)-\mathrm{A} \varepsilon_{\mathbf{y}}\left(\psi^{i j}\right) \cdot \varepsilon_{\mathbf{y}}\left(\xi^{k l m}\right)\right) \mathrm{d} \mathbf{y}, \\
\mathrm{C}_{i j k l m n}^{(0)} & =\int_{Y}\left(\mathrm{~A}\left(\chi^{i j} \odot \mathbf{e}_{k}\right) \cdot\left(\chi^{l m} \odot \mathbf{e}_{n}\right)-\mathrm{A} \varepsilon_{\mathbf{y}}\left(\boldsymbol{\xi}^{i j k}\right) \cdot \varepsilon_{\mathbf{y}}\left(\boldsymbol{\xi}^{l m n}\right)\right) \mathrm{d} \mathbf{y} .
\end{aligned}
$$


Note that one must use the elementary problems giving the $\chi^{i j \prime}, \psi^{i j \prime}$ s and $\xi^{k l m}$ to obtain the expressions above. As the three previous ones, these stiffness tensor fields depend only on the microstructure and admit the same type of symmetries. Specifically, one has

$$
\mathrm{A}_{i j k l}^{(2)}=\mathrm{A}_{i j l k}^{(2)}=\mathrm{A}_{k l i j}^{(2)}, \quad \mathrm{B}_{i j k l m}^{(1)}=\mathrm{B}_{j i k l m}^{(1)}=\mathrm{B}_{i j l k m}^{(1)}, \quad \mathrm{C}_{i j k l m n}^{(0)}=\mathrm{C}_{j i k l m n}^{(0)}=\mathrm{C}_{l m n i j k}^{(0)} .
$$

The properties of positivity of $A^{(2)}$ and $C^{(0)}$ do not appear clearly from the expressions above. In fact, their positivity is not guaranteed as we will se in the example developed in Section 4.

\subsubsection{Minimizing with respect to $\langle\mathbf{v}\rangle$}

Minimizing $\overline{\bar{J}}^{(2)}$ leads to the following variational equation for $\left\langle\mathbf{u}^{1}\right\rangle$

$$
\int_{\Omega}\left(\mathrm{A}^{(0)} \boldsymbol{\varepsilon}_{\mathbf{x}}\left(\left\langle\mathbf{u}^{1}\right\rangle\right)+\mathrm{A}^{(1)} \boldsymbol{\varepsilon}_{\mathbf{x}}\left(\mathbf{u}^{0}\right)+\mathrm{B}^{(0)} \mathbf{g}_{\mathbf{x}}\left(\mathbf{u}^{0}\right)\right) \cdot \boldsymbol{\varepsilon}_{\mathbf{x}}(\langle\mathbf{v}\rangle) \mathrm{d} \mathbf{x}=0, \quad \forall\langle\mathbf{v}\rangle \in H_{0}^{1}\left(\Omega, \mathbb{R}^{2}\right)
$$

which admits a unique solution under the conditions stated in Remark 3. Accordingly, $\left\langle\mathbf{u}^{1}\right\rangle$ is solution of the following linear elastic problem defined on the "homogenized" body which involves the three effective tensor fields $A^{(0)}, A^{(1)}$ and $B^{(0)}$

$$
\begin{cases}\operatorname{div}_{\mathbf{x}}\left(\mathrm{A}^{(\mathbf{0})} \varepsilon_{\mathbf{x}}\left(\left\langle\mathbf{u}^{1}\right\rangle\right)+\mathrm{A}^{(1)} \boldsymbol{\varepsilon}_{\mathbf{x}}\left(\mathbf{u}^{0}\right)+\mathrm{B}^{(0)} \mathbf{g}_{\mathbf{x}}\left(\mathbf{u}^{0}\right)\right)=\mathbf{0}, & \text { in } \Omega \\ \left\langle\mathbf{u}^{1}\right\rangle=0, & \text { on } \partial \Omega\end{cases}
$$

Once this problem is solved, $\mathbf{u}^{1}$ is entirely determined and we obtain from (47) that the third term $\mathbf{u}^{2}$ of the expansion of $\mathbf{u}^{\epsilon}$ can read as

$$
\mathbf{u}^{2}=\varepsilon_{\mathbf{x} p q}\left(\left\langle\mathbf{u}^{1}\right\rangle\right) \chi^{p q}+\varepsilon_{\mathbf{x} p q}\left(\mathbf{u}^{0}\right) \psi^{p q}+g_{\mathbf{x} p q r}\left(\mathbf{u}^{0}\right) \xi^{p q r}+\left\langle\mathbf{u}^{2}\right\rangle,
$$

its average value $\left\langle\mathbf{u}^{2}\right\rangle$ only remaining unknown at this stage. Moreover, the value of the energy $J_{*}^{(2)}=\overline{\bar{J}}^{(2)}\left(\left\langle\mathbf{u}^{1}\right\rangle\right)$ is obtained from (50)

$$
\begin{aligned}
J_{*}^{(2)} & =\int_{\Omega}\left(\frac{1}{2} \mathrm{~A}^{(0)} \varepsilon_{\mathbf{x}}\left(\left\langle\mathbf{u}^{1}\right\rangle\right) \cdot \varepsilon_{\mathbf{x}}\left(\left\langle\mathbf{u}^{1}\right\rangle\right)+\left(\mathrm{A}^{(1)} \varepsilon_{\mathbf{x}}\left(\mathbf{u}^{0}\right)+\mathrm{B}^{(0)} \mathbf{g}_{\mathbf{x}}\left(\mathbf{u}^{0}\right)\right) \cdot \varepsilon_{\mathbf{x}}\left(\left\langle\mathbf{u}^{1}\right\rangle\right)\right) \mathrm{d} \mathbf{x} \\
& +\int_{\Omega}\left(\frac{1}{2} \mathrm{~A}^{(2)} \varepsilon_{\mathbf{x}}\left(\mathbf{u}^{0}\right) \cdot \varepsilon_{\mathbf{x}}\left(\mathbf{u}^{0}\right)+\mathrm{B}^{(1)} \mathbf{g}_{\mathbf{x}}\left(\mathbf{u}^{0}\right) \cdot \varepsilon_{\mathbf{x}}\left(\mathbf{u}^{0}\right)+\frac{1}{2} \mathrm{C}^{(0)} \mathbf{g}_{\mathbf{x}}\left(\mathbf{u}^{0}\right) \cdot \mathbf{g}_{\mathbf{x}}\left(\mathbf{u}^{0}\right)\right) \mathrm{d} \mathbf{x},
\end{aligned}
$$

and the second order stress field $\sigma^{1}$ is also known,

$$
\sigma^{1}=\mathrm{A}\left(\varepsilon_{\mathbf{x}}\left(\mathbf{u}^{1}\right)+\varepsilon_{\mathbf{y}}\left(\mathbf{u}^{2}\right)\right) .
$$

\subsection{To summarize}

At this stage, we have obtained the first two terms of the expansion both for the displacements and the stresses, that is to say $\mathbf{u}^{0}, \mathbf{u}^{1}, \sigma^{0}$ and $\sigma^{1}$. The next term $\mathbf{u}^{2}$ of the displacement expansion is known up to its average value $\left\langle\mathbf{u}^{2}\right\rangle$. Moreover the first three terms $J_{*}^{(0)}, J_{*}^{(1)}$ and $J_{*}^{(2)}$ of the expansion of the energy at equilibrium $J^{\epsilon}\left(\mathbf{u}^{\epsilon}\right)$ are also 
known and we could prove that the next term $J_{*}^{(3)}$ is also determined. All these determinations require: (i) first, to solve three families of elastic problems posed on the current unit cell $\{\mathbf{x}\} \times Y$ whose solutions $\chi^{i j}, \psi^{i j}$ and $\xi^{i j k}$ (with $i, j$ and $k$ running in $\{1,2,3\}$ ) depend only on the microstructure at $\mathbf{x}$; (ii) then, to calculate the six effective tensor fields $A^{(0)}, A^{(1)}, A^{(2)}, B^{(0)}, B^{(1)}, C^{(0)}$ defined on the "homogenized" domain $\Omega$; (iii) then, to solve the two macroscopic elastic problems whose solutions are $\mathbf{u}^{0}$ and $\left\langle\mathbf{u}^{1}\right\rangle$; (iv) finally, the fields $\mathbf{u}^{0}, \mathbf{u}^{1}, \sigma^{0}$ and $\sigma^{1}$ are obtained by linear combinations of the previous ones and the energetic terms $J_{*}^{(0)}, J_{*}^{(1)}$ and $J_{*}^{(2)}$ are given by integral calculus. We could continue the procedure to obtain the next terms.

\section{AN ILLUSTRATIVE EXAMPLE}

To illustrate the procedure described in the previous sections, we consider the case of an heterogeneous cylinder made of a bi-layer laminate and submitted to the gravity. Specifically, the cylinder is $\Omega=S \times(0, H)$ with height $H$ and whose cross section $S$ is a bounded, connected open subset of $\mathbb{R}^{2}$ with boundary $\partial S$. This cylinder is made of a finely bi-layered composite, the two materials constituting the layers having the same density $\rho$ and being isotropically elastic with Lamé coefficients $\left(\lambda_{1}, \mu_{1}\right)$ and $\left(\lambda_{2}, \mu_{2}\right)$. The spatial distribution of the layers is such that the stiffness $\mathrm{A}^{\epsilon}$ depends on $\left(x_{1}, x_{2}\right)$ only and not on $x_{3}$,

$$
\mathrm{A}^{\epsilon}(\mathbf{x})_{i j k l}=\lambda^{\epsilon}\left(x_{1}, x_{2}\right) \delta_{i j} \delta_{k l}+\mu^{\epsilon}\left(x_{1}, x_{2}\right)\left(\delta_{i k} \delta_{j l}+\delta_{i l} \delta_{j k}\right) .
$$

The lateral boundary of the cylinder $\partial S \times(\mathbf{0}, H)$ is clamped whereas the sections $S \times\{\mathbf{0}\}$ and $S \times\{H\}$ cannot move in the transversal directions but are free to move in the axial direction. Specifically the boundary conditions read as

$$
\mathbf{u}^{\epsilon}=\mathbf{0} \text { on } \partial S \times(0, H), \quad\left\{\begin{array}{l}
u_{1}^{\epsilon}=u_{2}^{\epsilon}=0 \\
\sigma_{33}^{\epsilon}=0
\end{array} \quad \text { on } S \times\{0, H\}\right.
$$

This cylinder being submitted to the gravity $-g \mathbf{e}_{3}$, the body forces are $\mathbf{f}(\mathbf{x})=-\rho g \mathbf{e}_{3}$ and hence are uniform. Under these conditions, it is easy to show that the (exact) displacement at equilibrium is anti-plane:

$$
\mathbf{u}^{\epsilon}(\mathbf{x})=u_{3}^{\epsilon}\left(x_{1}, x_{2}\right) \mathbf{e}_{3} .
$$

Moreover, by linearity, its unique non-null component $u_{3}^{\epsilon}$ can read as

$$
u_{3}^{\epsilon}\left(x_{1}, x_{2}\right)=\rho g u^{\epsilon}\left(x_{1}, x_{2}\right),
$$

where $u^{\epsilon}$ is the unique solution of the following problem posed on the cross-section $S$

$$
\begin{cases}\operatorname{div}\left(\mu^{\epsilon} \nabla u^{\epsilon}\right)=1, & \text { in } S \\ u^{\epsilon}=0, & \text { on } \partial S\end{cases}
$$

From an energetic point of view, $u^{\epsilon}$ is solution of the following minimization problem

Variational formulation: Find $u^{\epsilon} \in H_{0}^{1}(S)$ which minimizes the energy functional $J^{\epsilon}$ over 
$H_{0}^{1}(S)$

$$
J^{\epsilon}\left(u^{\epsilon}\right)=\min _{v \in H_{0}^{1}(S)} J^{\epsilon}(v) \text { with } J^{\epsilon}(v)=\int_{S}\left(\frac{1}{2} \mu^{\epsilon} \nabla v \cdot \nabla v-v\right) \mathrm{d} S .
$$

Note that the solution depends only on the distribution of the shear modulus $\mu^{\epsilon}$ in the cross-section $S$. Concerning that distribution, one considers a quasi-periodic case characterized by the following assumption

$$
\mu^{\epsilon}\left(x_{1}, x_{2}\right)=\mu\left(x_{1}, x_{2}, \frac{x_{1}}{\epsilon}\right) \quad \text { with } \quad \mu\left(x_{1}, x_{2}, y\right)= \begin{cases}\mu_{1} & \text { if } 2|y|<\theta\left(x_{1}, x_{2}\right) \\ \mu_{2} & \text { if } \quad \theta\left(x_{1}, x_{2}\right)<2|y|<1\end{cases}
$$

where $\theta$ is a smooth function of $\left(x_{1}, x_{2}\right)$ with values in $[0,1]$ and $\mu$ is periodic with respect to $y$ with period 1 . In other words, the two materials are layered in the direction 1 , in proportion $\theta$ for the material 1 and $1-\theta$ for the material 2, that proportion changing smoothly in the cross-section. So, we are in a situation corresponding to what is studied in the previous sections and even a little simpler. Indeed, here the body forces are constant, the unit cell is one-dimensional and corresponds to the interval $Y=(-1 / 2,+1 / 2)$, the quasi-periodic repartition of the heterogeneities is characterized by the scalar function $\theta$, the unknown is a scalar field and the domain $S$ is two-dimensional. In order to prevent boundary layer effects, it suffices to assume that $\theta$ is equal to 0 (or equivalently to 1 ) in a neighborhood of $\partial S$. We can follow the procedure described above to find the first terms of the expansion of the solution with respect to $\epsilon$. Throughout this section, the Greek indices $\alpha, \beta, \gamma, \ldots$ run in $\{1,2\}$.

\subsection{Determination of $\chi^{\alpha 3}, a_{\alpha 3 \beta 3}^{0}, A_{\alpha 3 \beta 3}^{(0)}, A_{\alpha 3 \beta 3}^{(1)}$ and $B_{\alpha 3 \beta 3 \gamma}^{(0)}$}

Here all the displacement fields have only their third component which is not identically zero and hence can be considered as scalar functions defined on $S \times(-1 / 2,1 / 2)$

$$
\chi^{i j}(\mathbf{x}, \mathbf{y})=\chi^{i j}\left(x_{1}, x_{2}, y\right) \mathbf{e}_{3} .
$$

Furthermore, $\chi^{\alpha 3}=\chi^{3 \alpha}$ only are to be determined for $\alpha=1$, 2. Starting from (20) and using the one-dimensional character of the unit cell, $\chi^{13}$ and $\chi^{23}$ are $Y$-periodic and such that

$$
\left\{\begin{array}{l}
\partial_{y}\left(\mu\left(\partial_{y} \chi^{13}+1\right)\right)=0 \\
\left\langle\chi^{13}\right\rangle=0
\end{array}, \quad\left\{\begin{array}{l}
\partial_{y}\left(\mu\left(\partial_{y} \chi^{23}\right)\right)=0 \\
\left\langle\chi^{23}\right\rangle=0
\end{array}\right.\right.
$$

One easily deduces that $\chi^{23}=0$ and that $\chi^{13}$ is the following piecewise linear and odd function of $y$ with slopes depending on $\mathbf{x}:=\left(x_{1}, x_{2}\right)$, see Fig. 2

$$
\chi^{13}(\mathbf{x}, y)=\frac{\left(\mu_{2}-\mu_{1}\right)}{m(\theta(\mathbf{x}))}\left\{\begin{array}{lll}
(1-\theta(\mathbf{x})) y & \text { when } & 2|y| \leq \theta(\mathbf{x}) \\
\theta(\mathbf{x})\left(\frac{\operatorname{sign}(y)}{2}-y\right) & \text { when } & \theta(\mathbf{x}) \leq 2|y| \leq 1
\end{array}\right.
$$

where

$$
\mathrm{m}(\theta):=(1-\theta) \mu_{1}+\theta \mu_{2}
$$

Note that $\chi^{13}=0$ in the homogeneous cases, i.e. when $\mu_{1}=\mu_{2}$ or $\theta \in\{0,1\}$. 


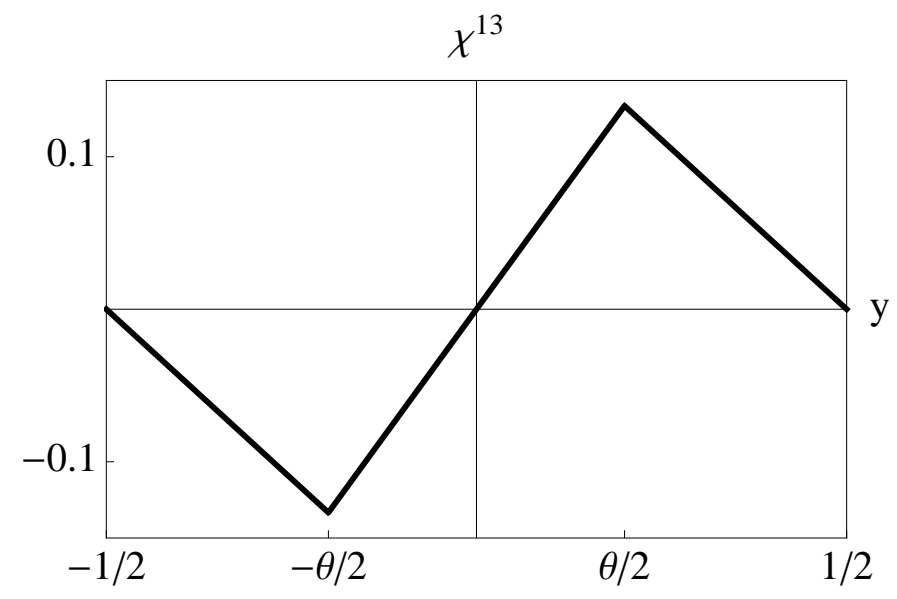

Fig. 2. Graph of $\chi^{13}$ when $\theta=0.4$ and $\mu_{2}=3 \mu_{1}$

We will use also the derivatives $\partial_{x_{\alpha}} \chi^{13}$ of $\chi^{13}$ which read as

$$
\partial_{x_{\alpha}} \chi^{13}(\mathbf{x}, y)=\frac{\left(\mu_{2}-\mu_{1}\right)}{m(\theta(\mathbf{x}))^{2}} \partial_{x_{\alpha}} \theta(\mathbf{x}) \begin{cases}-\mu_{2} y & \text { when } 2|y|<\theta(\mathbf{x}) \\ \mu_{1}\left(\frac{\operatorname{sign}(y)}{2}-y\right) & \text { when } \quad \theta(\mathbf{x})<2|y|<1\end{cases}
$$

Note that they are discontinuous at $y= \pm \theta(\mathbf{x}) / 2$ and that they are proportional to the gradient of $\theta$. Note also that they are identically null when $\mu_{1}=\mu_{2}$, but not when $\theta(\mathbf{x})=$ 0 or 1 and $\partial_{x_{\alpha}} \theta(\mathbf{x}) \neq 0$.

Using (30), one obtains the components $a_{\alpha 3 \beta 3}^{0}$ (and those which are deduced by symmetry) of the stress localization tensor field:

$$
a_{1313}^{0}(\mathbf{x}, y)=\frac{\mu_{1} \mu_{2}}{\mathrm{~m}(\theta(\mathbf{x}))}, \quad a_{1323}^{0}(\mathbf{x}, y)=a_{2313}^{0}(\mathbf{x}, y)=0, \quad a_{2323}^{0}(\mathbf{x}, y)=\mu(\mathbf{x}, y)
$$

Then, their average value over $Y$ gives the components of the effective stiffness tensor $\mathrm{A}^{(0)}$

$$
A_{1313}^{(0)}(\mathbf{x})=\frac{\mu_{1} \mu_{2}}{m(\theta(\mathbf{x}))}, \quad A_{1323}^{(0)}(\mathbf{x})=A_{2313}^{(0)}(\mathbf{x})=0, \quad A_{2323}^{(0)}(\mathbf{x})=\theta(\mathbf{x}) \mu_{1}+(1-\theta(\mathbf{x})) \mu_{2},
$$

which bring the harmonic mean value $1 /\langle 1 / \mu\rangle$ and the arithmetic mean value $\langle\mu\rangle$ of the shear modulus into play. Since $\chi^{13}$ is an odd function of $y$ and since $\chi^{23}$ vanishes, it is easy to deduce from (37) and (67) that all the relevant components of $B^{(0)}$ vanish

$$
\mathrm{B}_{\alpha 3 \beta 3 \gamma}^{(0)}=\mathrm{B}_{3 \alpha \beta 3 \gamma}^{(0)}=\mathrm{B}_{\alpha 33 \beta \gamma}^{(0)}=\mathrm{B}_{3 \alpha 3 \beta \gamma}^{(0)}=0, \quad \forall \alpha, \beta, \gamma \in\{1,2\}
$$

The relevant components of $A^{(1)}$ are $A_{\alpha 3 \beta 3}^{(1)}$ (and those deduced by symmetry). From (37) they read $A_{\alpha 3 \beta 3}^{(1)}=-\left\langle\partial_{x_{\gamma}}\left(a_{\gamma 3 \beta 3}^{0}\right) \chi^{\alpha 3}\right\rangle$. Since $\chi^{23}=0$, one immediately obtains $A_{2313}^{(1)}=$ 
$A_{2323}^{(1)}=0$. Using (67) and noting that $a_{1313}^{0}$ does not depend on $y, A_{1313}^{(1)}$ becomes

$$
\mathrm{A}_{1313}^{(1)}=-\left\langle\partial_{x_{1}}\left(\mathrm{a}_{1313}^{0}\right) \chi^{13}\right\rangle=-\partial_{x_{1}}\left(\mathrm{a}_{1313}^{0}\right)\left\langle\chi^{13}\right\rangle=0
$$

The calculation of $A_{1323}^{(1)}$ requires some attention. Indeed, by (37) and (67), $A_{1323}^{(1)}=$ $-\left\langle\chi^{13} \partial_{x_{2}} \mu\right\rangle$ which brings the spatial derivative of $\mu$ into play. But since $\mu$ is piecewise constant, that derivative must be understood in a weak sense. Since $\left\langle\mu \chi^{13}\right\rangle=0$ by symmetry, one must read in fact $A_{1323}^{(1)}=\left\langle\mu \partial_{x_{2}} \chi^{13}\right\rangle$ where the derivative of $\chi^{13}$ can be considered in the classical sense because $\chi^{13}$ is continuous and piecewise differentiable in $S \times Y$. Accordingly, since $\partial_{x_{2}} \chi^{13}$ is an odd function of $y$ and $\mu$ is an even function of $y$, one finally gets $A_{1323}^{(1)}=0$. Therefore, all the relevant components of $A^{(1)}$ in the present anti-plane context vanish.

\subsection{Determination of $u^{0}, \sigma_{\alpha 3}^{0},\left\langle u^{1}\right\rangle, J_{*}^{(0)}, J_{*}^{(1)}$}

The first term $u^{0}$ of the expansion of $u^{\epsilon}$ depends on $\left(x_{1}, x_{2}\right)$ only and is the unique solution of the following linear problem posed on the "homogenized" cross-section

$$
\begin{cases}\partial_{x_{1}}\left(\langle 1 / \mu\rangle^{-1} \partial_{x_{1}} u^{0}\right)+\partial_{x_{2}}\left(\langle\mu\rangle \partial_{x_{2}} u^{0}\right)=1 & \text { in } S \\ u^{0}=0 & \text { on } \quad \partial S\end{cases}
$$

Therefore $u^{0}$ depends only on the repartition of the proportion $\left(x_{1}, x_{2}\right) \mapsto \theta\left(x_{1}, x_{2}\right)$ of the material 1 in the section $S$. Moreover $u^{0}$ minimizes $\bar{J}^{(0)}$ over $H_{0}^{1}(S)$ with $\bar{J}^{(0)}$ given by

$$
\bar{J}^{(0)}(v)=\frac{1}{2} \int_{S}\left(\frac{1}{\langle 1 / \mu\rangle} \partial_{x_{1}} v \partial_{x_{1}} v+\langle\mu\rangle \partial_{x_{2}} v \partial_{x_{2}} v\right) \mathrm{d} S-\int_{S} v \mathrm{~d} S .
$$

By standard arguments, on deduces that the minimum is given by

$$
J_{*}^{(0)}:=\bar{J}^{(0)}\left(u^{0}\right)=-\frac{1}{2} \int_{S} u^{0} \mathrm{~d} S .
$$

Once $u^{0}$ is determined, the first order stress field $\sigma^{0}$ is obtained from (29) and (67)

$$
\sigma_{13}^{0}(\mathbf{x})=\frac{1}{\langle 1 / \mu\rangle(\mathbf{x})} \partial_{x_{1}} u^{0}(\mathbf{x}), \quad \sigma_{23}^{0}(\mathbf{x}, y)=\mu(\mathbf{x}, y) \partial_{x_{2}} u^{0}(\mathbf{x}) .
$$

Since all the relevant components of $A^{(1)}$ and $B^{(0)}$ vanish, one immediately deduces from (36) and (55) that

$$
\left\langle u^{1}\right\rangle=0, \quad J_{*}^{(1)}=0 .
$$

Therefore $u^{1}$ is given by

$$
u^{1}(\mathbf{x}, y)=\partial_{x_{1}} u^{0}(\mathbf{x}) \chi^{13}(\mathbf{x}, y) .
$$

4.3. Determination of $\psi^{\alpha 3}, \xi^{\alpha 3 \beta}, \mathrm{A}_{\alpha 3 \beta 3}^{(2)}, \mathrm{B}_{\alpha 3 \beta 3 \gamma}^{(1)}, \mathrm{C}_{\alpha 3 \beta \gamma 3 \zeta}^{(0)}$ and $J_{*}^{(2)}$

The goal of this subsection is to obtain $J_{*}^{(2)}$, i.e. the term of the order of $\epsilon^{2}$ in the energy expansion. For that, one must solve the other elementary problems and calculate the other effective stiffness tensors. 


\subsubsection{Determination of $\psi^{\alpha 3}$ and $\xi^{\alpha 3 \beta}$}

Let us first note that the effective stiffness tensors $A^{(2)}, B^{(1)}$ and $C^{(0)}$ bring into play the gradients with respect to $y$ of the fields $\psi^{\alpha 3}, \xi^{\alpha 3 \beta}$ only, see (51)-(53). Therefore, it is sufficient to determine $\partial_{y} \psi^{\alpha 3}$ and $\partial_{y} \xi^{\alpha 3 \beta}$, leaving undetermined the constant of integration (which could be obtained by the conditions $\left\langle\psi^{\alpha 3}\right\rangle=\left\langle\xi^{\alpha 3 \beta}\right\rangle=0$ ).

From (48) and (67), one sees that $\psi^{13}$ must satisfy

$$
\left\langle\mu\left(\partial_{y} \psi^{13}+\partial_{x_{1}} \chi^{13}\right) \partial_{y} \phi\right\rangle=\left\langle\partial_{x_{1}} a_{1313}^{0} \phi\right\rangle, \quad \forall \phi \in H_{\#}^{1}(S),\langle\phi\rangle=0 .
$$

But since $a_{1313}^{0}$ does not depend on $y$ and $\langle\phi\rangle=0$, the right hand side of the above variational equality vanishes and hence $\left\langle\mu\left(\partial_{y} \psi^{13}+\partial_{x_{1}} \chi^{13}\right) \partial_{y} \phi\right\rangle=0$ for all $\phi \in H_{\#}^{1}(S)$ such that $\langle\phi\rangle=0$. Therefore $\mu\left(\partial_{y} \psi^{13}+\partial_{x_{1}} \chi^{13}\right)$ does not depend on $y$ and one gets

$$
\partial_{y} \psi^{13}(\mathbf{x}, y)+\partial_{x_{1}} \chi^{13}(\mathbf{x}, y)=\frac{a(\mathbf{x})}{\mu(\mathbf{x}, y)},
$$

with the constant $a(\mathbf{x})$ to be determined. But since $\chi^{13}$ is $Y$-periodic and since $\chi^{13}$ is an odd function of $y$, one has $\left\langle\partial_{y} \psi^{13}\right\rangle=\left\langle\partial_{x_{1}} \chi^{13}\right\rangle=0$. Hence (76) gives $a(\mathbf{x})=0$ and one finally obtains

$$
\partial_{y} \psi^{13}=-\partial_{x_{1}} \chi^{13},\left\langle\psi^{13}\right\rangle=0,
$$

which determines $\psi^{13}$ with the help of (64).

The determination of $\psi^{23}$ requires some attention. Indeed, since $\chi^{23}=0$, using (48) and (67), $\psi^{23}$ should satisfy

$$
\left\langle\mu \partial_{y} \psi^{23} \partial_{y} \phi\right\rangle=\left\langle\phi \partial_{x_{2}} \mu\right\rangle, \quad \forall \phi \in H_{\#}^{1}(S),\langle\phi\rangle=0
$$

But since $\mu$ is piecewise constant, the derivative of $\mu$ must be understood in a weak sense. Specifically, let us consider that $\left\langle\phi \partial_{x_{2}} \mu\right\rangle$ is a distribution, i.e. an element of the dual $\mathcal{D}^{\prime}(S)$ of $\mathcal{D}(S)$. Then $\left\langle\phi \partial_{x_{2}} \mu\right\rangle$ is defined by

$$
\left\langle\phi \partial_{x_{2}} \mu\right\rangle(\varphi)=-\int_{S \times Y} \mu(\mathbf{x}, y) \phi(y) \partial_{x_{2}} \varphi(\mathbf{x}) \mathrm{d} y \mathrm{~d} S, \quad \forall \varphi \in \mathcal{D}(S) .
$$

By Fubini theorem, one gets

$$
\left\langle\phi \partial_{x_{2}} \mu\right\rangle(\varphi)=-\int_{S}\left(\mu_{1} \int_{2|y|<\theta(\mathbf{x})} \phi(y) \mathrm{d} y+\mu_{2} \int_{\theta(\mathbf{x})<2|y|<1} \phi(y) \mathrm{d} y\right) \partial_{x_{2}} \varphi(\mathbf{x}) \mathrm{d} S .
$$

Now, owing to the assumed regularity of $\mathbf{x} \mapsto \theta(\mathbf{x})$, one can make the integration by parts with respect to $x_{2}$ to obtain

$$
\left\langle\phi \partial_{x_{2}} \mu\right\rangle(\varphi)=-\int_{S} \frac{1}{2}\left(\mu_{2}-\mu_{1}\right) \partial_{x_{2}} \theta(\mathbf{x})(\phi(-\theta(\mathbf{x}) / 2)+\phi(+\theta(\mathbf{x}) / 2)) \varphi(\mathbf{x}) \mathrm{d} S .
$$

Therefore $\psi^{23}$ must in fact satisfy the following variational equation (where the dependence on $\mathbf{x}$ is not indicated)

$$
\int_{-1 / 2}^{1 / 2} \mu \partial_{y} \psi^{23} \partial_{y} \phi \mathrm{d} y=-\frac{1}{2}\left(\mu_{2}-\mu_{1}\right) \partial_{x_{2}} \theta(\phi(-\theta / 2)+\phi(+\theta / 2)),
$$


for all $\phi \in H_{\#}^{1}(S)$ with $\langle\phi\rangle=0$. It can be solved in a closed form and after some calculations left to the reader one finally gets

$$
\partial_{y} \psi^{23}(\mathbf{x}, y)=\left(\mu_{1}-\mu_{2}\right) \partial_{x_{2}} \theta(\mathbf{x})\left\{\begin{array}{lll}
\frac{y}{\mu_{1}} & \text { when } \quad 2|y|<\theta(\mathbf{x}) \\
\frac{y}{\mu_{2}}-\frac{\operatorname{sign}(y)}{2 \mu_{2}} & \text { when } \quad \theta(\mathbf{x})<2|y|<1
\end{array}\right.
$$

That determines $\psi^{23}$ up to a constant which is fixed by the condition $\left\langle\psi^{23}\right\rangle=0$.

From (49) and (67), one gets that $\xi^{131}$ is the unique solution of

$$
\left\{\begin{array}{l}
\xi^{131} \in H_{\#}^{1}(S),\left\langle\xi^{131}\right\rangle=0 \\
\left\langle\mu\left(\partial_{y} \xi^{131}+\chi^{13}\right) \partial_{y} \phi\right\rangle=0, \quad \forall \phi \in H_{\#}^{1}(S),\langle\phi\rangle=0 .
\end{array}\right.
$$

Let us verify that $\xi^{131}$ given by

$$
\partial_{y} \xi^{131}=-\chi^{13},\left\langle\xi^{131}\right\rangle=0,
$$

is solution. Indeed, $\xi^{131}$ given by (80) verifies the variational equation above. From $\left\langle\chi^{13}\right\rangle=0$, one deduces that $\left\langle\partial_{y} \xi^{131}\right\rangle=0$ and hence that $\xi^{131}$ is $Y$-periodic. The relation $\partial_{y} \xi^{131}=-\chi^{13}$ determines $\xi^{131}$ up to a constant which is fixed by the condition $\left\langle\xi^{131}\right\rangle=0$.

In a same manner, one gets from (49) and (67) that $\xi^{132}=\xi^{231}=0$. It remains to find $\xi^{232}$ which is the unique solution of

$$
\left\{\begin{array}{l}
\xi^{232} \in H_{\#}^{1}(S),\left\langle\xi^{232}\right\rangle=0, \\
\left\langle\mu \partial_{y} \xi^{232} \partial_{y} \phi\right\rangle=\langle\mu \phi\rangle, \quad \forall \phi \in H_{\#}^{1}(S),\langle\phi\rangle=0 .
\end{array}\right.
$$

It can be solved in a closed form and after some calculations left to the reader one finally gets

$$
\partial_{y} \xi^{232}(\mathbf{x}, y)=\left(\mu_{2}-\mu_{1}\right)\left\{\begin{array}{lll}
\frac{1-\theta}{\mu_{1}} y & \text { when } & 2|y|<\theta(\mathbf{x}) \\
\frac{\theta}{\mu_{2}}\left(\frac{\operatorname{sign}(y)}{2}-y\right) & \text { when } & \theta(\mathbf{x})<2|y|<1
\end{array}\right.
$$

That determines $\xi^{232}$ up to a constant which is fixed by the condition $\left\langle\xi^{232}\right\rangle=0$. Let us note that the displacement fields $\psi^{13}, \psi^{23}, \xi^{131}$ and $\xi^{232}$ enjoy the following properties:

(1) They are identically null when the medium is homogeneous, i.e. when $\theta \in$ $\{0,1\}$ everywhere or when $\mu_{1}=\mu_{2}$, but $\psi^{13}(\mathbf{x})$ or $\psi^{23}(\mathbf{x})$ are not identically null when the medium is only locally homogenous at $\mathbf{x}$ with $\theta(\mathbf{x}) \in\{0,1\}$ and $\left(\partial_{x_{1}} \theta(\mathbf{x}), \partial_{x_{2}} \theta(\mathbf{x})\right) \neq(0,0)$.

(2) They are even, periodic and piecewise quadratic functions of $y$, see Fig. 3.

4.3.2. Calculation of $\mathrm{A}_{\alpha 3 \beta 3}^{(2)}, \mathrm{B}_{\alpha 3 \beta 3 \gamma}^{(1)}$ and $\mathrm{C}_{\alpha 3 \beta \gamma 3 \zeta}^{(0)}$

Using (51)-(53), the relevant components of these effective stiffness tensors read as

$$
\mathrm{A}_{\alpha 3 \beta 3}^{(2)}=\left\langle\mu \partial_{x_{\zeta}} \chi^{\alpha 3} \partial_{x_{\zeta}} \chi^{\beta 3}-\mu \partial_{y} \psi^{\alpha 3} \partial_{y} \psi^{\beta 3}\right\rangle,
$$



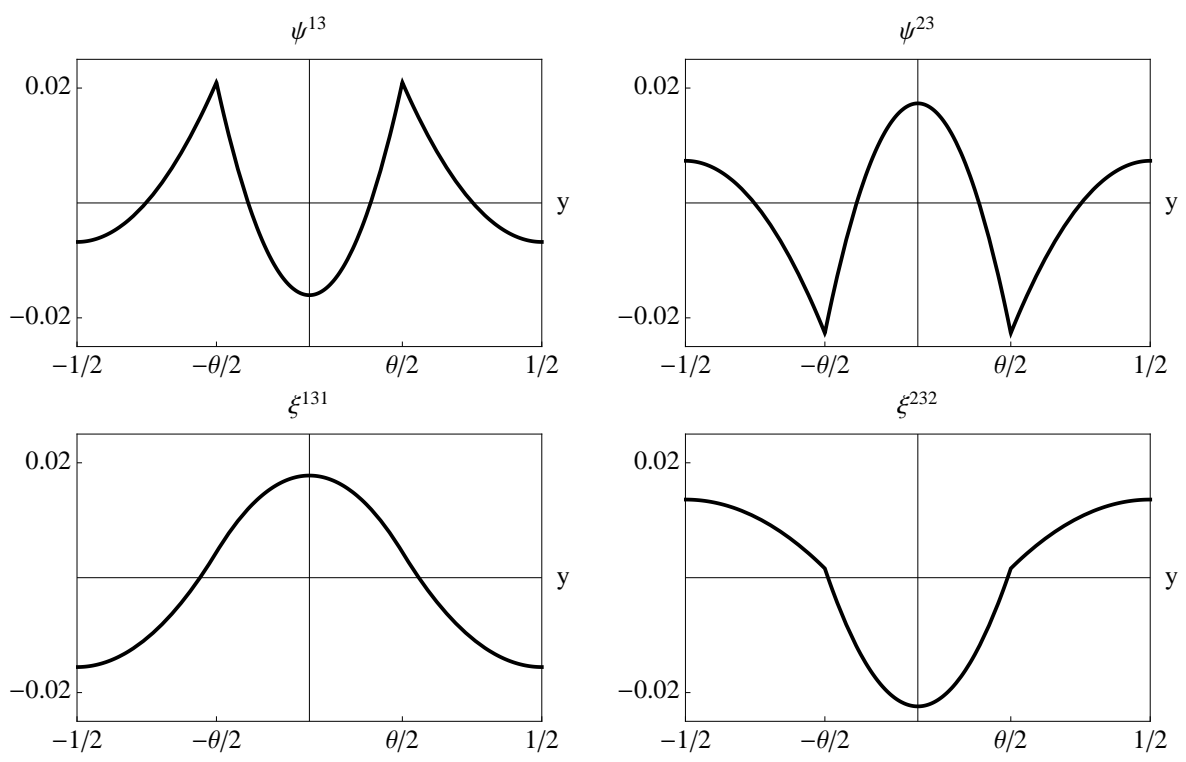

Fig. 3. Graph of $\psi^{13}, \psi^{23}, \xi^{131}$ and $\xi^{232}$ when $\theta=0.4, \mu_{2}=3 \mu_{1}$ and $\partial_{x_{1}} \theta=\partial_{x_{2}} \theta=1$

$$
\begin{gathered}
\mathrm{B}_{\alpha 3 \beta 3 \gamma}^{(1)}=\left\langle\mu \partial_{x_{\gamma}} \chi^{\alpha 3} \chi^{\beta 3}-\mu \partial_{y} \psi^{\alpha 3} \partial_{y} \xi^{\beta 3 \gamma}\right\rangle, \\
\mathrm{C}_{\alpha 3 \beta \gamma 3 \zeta}^{(0)}=\left\langle\mu \chi^{\alpha 3} \chi^{\gamma 3} \delta_{\beta \zeta}-\mu \partial_{y} \xi^{\alpha 3 \beta} \partial_{y} \xi^{\gamma 3 \zeta}\right\rangle,
\end{gathered}
$$

where $\delta$ denotes the Kronecker symbol. Since all the elementary fields are known, it suffices to calculate the integrals over $Y$. Using (66), (77), (79) and (82), one gets for $A^{(2)}$

$$
\begin{gathered}
\mathrm{A}_{1313}^{(2)}=\left\langle\mu \partial_{x_{2}} \chi^{13} \partial_{x_{2}} \chi^{13}\right\rangle=\frac{\left(\mu_{2}-\mu_{1}\right)^{2} \mu_{1} \mu_{2}\left((1-\theta)^{3} \mu_{1}+\theta^{3} \mu_{2}\right)}{12\left((1-\theta) \mu_{1}+\theta \mu_{2}\right)^{4}}\left(\partial_{x_{2}} \theta\right)^{2}, \\
\mathrm{~A}_{1323}^{(2)}=\left\langle\mu \partial_{x_{1}} \chi^{13} \partial_{y} \psi^{23}\right\rangle=\frac{\left(\mu_{2}-\mu_{1}\right)^{2}\left((1-\theta)^{3} \mu_{1}+\theta^{3} \mu_{2}\right)}{12\left((1-\theta) \mu_{1}+\theta \mu_{2}\right)^{2}} \partial_{x_{1}} \theta \partial_{x_{2}} \theta \\
\mathrm{A}_{2323}^{(2)}=-\left\langle\mu \partial_{y} \psi^{23} \partial_{y} \psi^{23}\right\rangle=-\frac{\left(\mu_{2}-\mu_{1}\right)^{2}}{12 \mu_{1} \mu_{2}}\left((1-\theta)^{3} \mu_{1}+\theta^{3} \mu_{2}\right)\left(\partial_{x_{2}} \theta\right)^{2}
\end{gathered}
$$

For $\mathrm{B}^{(1)}$, using (83) and the expressions for the elementary fields, one gets

$$
\begin{gathered}
\mathrm{B}_{13131}^{(1)}=\mathrm{B}_{13231}^{(1)}=\mathrm{B}_{23132}^{(1)}=0, \\
\mathrm{~B}_{13132}^{(1)}=\left\langle\mu \chi^{13} \partial_{x_{2}} \chi^{13}\right\rangle=\frac{\left(\mu_{2}-\mu_{1}\right)^{2} \mu_{1} \mu_{2} \theta(1-\theta)(1-2 \theta)}{12\left((1-\theta) \mu_{1}+\theta \mu_{2}\right)^{3}} \partial_{x_{2}} \theta, \\
\mathrm{B}_{23131}^{(1)}=\left\langle\mu \chi^{13} \partial_{y} \psi^{23}\right\rangle=\frac{\left(\mu_{2}-\mu_{1}\right)^{2} \theta(1-\theta)(1-2 \theta)}{12\left((1-\theta) \mu_{1}+\theta \mu_{2}\right)} \partial_{x_{2}} \theta,
\end{gathered}
$$




$$
\begin{array}{r}
\mathrm{B}_{13232}^{(1)}=\left\langle\mu \partial_{x_{1}} \chi^{13} \partial_{y} \xi^{232}\right\rangle=\frac{\left(\mu_{2}-\mu_{1}\right)^{2} \theta(1-\theta)\left((1-\theta)^{2} \mu_{1}-\theta^{2} \mu_{2}\right)}{12\left((1-\theta) \mu_{1}+\theta \mu_{2}\right)^{2}} \partial_{x_{1}} \theta, \\
\mathrm{B}_{23232}^{(1)}=-\left\langle\mu \partial_{y} \psi^{23} \partial_{y} \xi^{232}\right\rangle=-\frac{\left(\mu_{2}-\mu_{1}\right)^{2} \theta(1-\theta)\left((1-\theta)^{2} \mu_{1}-\theta^{2} \mu_{2}\right)}{12 \mu_{1} \mu_{2}} \partial_{x_{2}} \theta .
\end{array}
$$

For $\mathrm{C}^{(0)}$, using (84) and the expressions for the elementary fields, one gets that the unique non null components are

$$
\begin{gathered}
\mathrm{C}_{131232}^{(0)}=\left\langle\mu \chi^{13} \partial_{y} \xi^{232}\right\rangle=\frac{\left(\mu_{2}-\mu_{1}\right)^{2} \theta^{2}(1-\theta)^{2}}{12\left((1-\theta) \mu_{1}+\theta \mu_{2}\right)}, \\
\mathrm{C}_{132132}^{(0)}=\left\langle\mu \chi^{13} \chi^{13}\right\rangle=\frac{\left(\mu_{2}-\mu_{1}\right)^{2} \theta^{2}(1-\theta)^{2}\left(\theta \mu_{1}+(1-\theta) \mu_{2}\right)}{12\left((1-\theta) \mu_{1}+\theta \mu_{2}\right)^{2}}, \\
\mathrm{C}_{232232}^{(0)}=-\left\langle\mu \partial_{y} \xi^{232} \partial_{y} \xi^{232}\right\rangle=-\frac{\left(\mu_{2}-\mu_{1}\right)^{2}}{12 \mu_{1} \mu_{2}} \theta^{2}(1-\theta)^{2}\left((1-\theta) \mu_{1}+\theta \mu_{2}\right),
\end{gathered}
$$

and those which are obtained by symmetry.

\subsubsection{Calculation of $J_{*}^{(2)}$}

Inserting the expressions above into (57) gives the term of order $\epsilon^{2}$ in the expansion of the energy,

$$
\begin{aligned}
J_{*}^{(2)} & =\int_{S} \frac{1}{2}\left(\mathrm{~A}_{1313}^{(2)} \partial_{1} u^{0} \partial_{1} u^{0}+2 \mathrm{~A}_{1323}^{(2)} \partial_{1} u^{0} \partial_{2} u^{0}+\mathrm{A}_{2323}^{(2)} \partial_{2} u^{0} \partial_{2} u^{0}\right) \mathrm{d} S \\
& +\int_{S}\left(\mathrm{~B}_{13132}^{(1)} \partial_{1} u^{0} \partial_{12}^{2} u^{0}+\mathrm{B}_{13232}^{(1)} \partial_{1} u^{0} \partial_{22}^{2} u^{0}+\mathrm{B}_{23131}^{(1)} \partial_{2} u^{0} \partial_{11}^{2} u^{0}+\mathrm{B}_{23232}^{(1)} \partial_{2} u^{0} \partial_{22}^{2} u^{0}\right) \mathrm{d} S \\
& +\int_{S} \frac{1}{2}\left(C_{132132}^{2} \partial_{12}^{2} u^{0} \partial_{12}^{2} u^{0}+2 C_{131232}^{(0)} \partial_{11}^{2} u^{0} \partial_{22}^{2} u^{0}+C_{232232}^{(0)} \partial_{22}^{2} u^{0} \partial_{22}^{2} u^{0}\right) \mathrm{d} S,
\end{aligned}
$$

where $\partial_{\alpha} \mathbf{u}^{0}$ and $\partial_{\alpha \beta}^{2} u^{0}$ stand for $\partial_{x_{\alpha}} u^{0}$ and $\partial_{x_{\alpha}}\left(\partial_{x_{\beta}} u^{0}\right)$, respectively. Since the components of $A^{(2)}$ and $B^{(1)}$ contain the gradient of $\theta$, it is more convenient to render this dependence explicit by expressing $J_{*}^{(2)}$ as

$$
\begin{aligned}
J_{*}^{(2)} & =\int_{S} \frac{1}{2}\left(a_{11}\left(\partial_{1} \theta \partial_{1} u^{0}\right)^{2}+2 a_{12} \partial_{1} \theta \partial_{1} u^{0} \partial_{2} \theta \partial_{2} u^{0}+a_{22}\left(\partial_{2} \theta \partial_{2} u^{0}\right)^{2}\right) \mathrm{d} S \\
& +\int_{S}\left(b_{112} \partial_{2} \theta \partial_{1} u^{0} \partial_{12}^{2} u^{0}+b_{122} \partial_{1} \theta \partial_{1} u^{0} \partial_{22}^{2} u^{0}+b_{211} \partial_{2} \theta \partial_{2} u^{0} \partial_{11}^{2} u^{0}+b_{222} \partial_{2} \theta \partial_{2} u^{0} \partial_{22}^{2} u^{0}\right) \mathrm{d} S \\
& +\int_{S} \frac{1}{2}\left(c_{1212} \partial_{12}^{2} u^{0} \partial_{12}^{2} u^{0}+2 c_{1122} \partial_{11}^{2} u^{0} \partial_{22}^{2} u^{0}+c_{2222} \partial_{22}^{2} u^{0} \partial_{22}^{2} u^{0}\right) \mathrm{d} S
\end{aligned}
$$

In (96) the coefficients $a_{\alpha \beta}, b_{\alpha \beta \gamma}$ and $c_{\alpha \beta \gamma \zeta}$ depend only on $\theta$ and their expression can be easily obtained from (85)-(95).

Remark 5. The second order energy term depends both on the second gradient of the displacement and on the gradient of the characteristic parameter of the microstructure. It turns out that the coefficients entering in the expression of this energy term have diffe rent signs: for instance, $\mathrm{A}_{1313}^{(2)}, \mathrm{C}_{131232}^{(0)}$ and $\mathrm{C}_{132132}^{(0)}$ are positive whereas $\mathrm{A}_{2323}^{(2)}$ and $\mathrm{C}_{232232}^{(0)}$ are negative. 


\section{CONCLUSION}

In this paper, we developed a homogenization procedure based on the combination of asymptotic expansions and a variational approach in order to construct the higher order macroscopic energy of a quasi-periodic heterogeneous medium. The main assumption used in this approach to describe the quasi-periodicity is that the properties of a point in the cell depends not only on its position but also the position of the cell in the medium. By using the asymptotic expansion of the strain energy, the minimization problem of energy microscopic becomes a series of successive minimization problems. The solutions of each problems give the corresponding macroscopic elastic energies, the components of these higher-order elastic energies are obtained by solving the cell problems where the solutions of previous orders become the entries for the next orders. We have shown that the effective energy density depends on the strain gradient and on the gradient of the microstructure as of the second order.

Concerning the perspectives open by this work and what extensions could be investigated, let us mention the following ones:

- The method could be apply to obtain the effective thermo-mechanical properties of thermo-elastic quasi-periodic media.

- It would be interesting to apply the same procedure for more general quasi-periodic media like those mentioned in Remark 1.

- The method was applied here by assuming that the microstructure is given (and fixed). It would be interesting to remove this assumption by considering that the microstructure depends on parameters that one has to optimize. For instance, we could extend the study made in Section 4 by considering that the proportion $\theta$ of the material 1 (which can be considered as the damaged material if $\mu_{1}<\mu_{2}$ ) is governed by a principle of least energy like in [17].

- Another possible application would be to consider micro-cracked media where the length and the orientation of the micro-cracks vary smoothly in the domain. We could so justify some models of damage which are regularized by introducing gradient damage terms like in [18-20].

- The analysis was made here by considering situations where there is no boundary layer effects. Since these situations are more the exception than the rule, it would be important to extend our study to the cases where boundary layer effects exist.

\section{REFERENCES}

[1] A. Bensoussan, J. L. Lions, and G. Papanicolaou. Asymptotic analysis of periodic structures, Vol. 374. Elsevier Science, (1978).

[2] F. Murat and L. Tartar. Calcul des variations et homogénéisation. Les méthodes del'homogénéisation: théorie et applications en physique, 57, (1985), pp. 319-369.

[3] G. Allaire. Homogenization and two-scale convergence. SIAM Journal on Mathematical Analysis, 23, (6), (1992), pp. 1482-1518. https://doi.org/10.1137/0523084.

[4] H. Dumontet. Study of a boundary layer problem in elastic composite materials. ESAIM: Mathematical Modelling and Numerical Analysis, 20, (2), (1986), pp. 265-286. https://doi.org/10.1051/m2an/1986200202651. 
[5] G. A. Francfort and F. Murat. Homogenization and optimal bounds in linear elasticity. Archive for Rational Mechanics and Analysis, 94, (4), (1986), pp. 307-334. https://doi.org/10.1007/bf00280908.

[6] R. Abdelmoula and J. J. Marigo. The effective behavior of a fiber bridged crack. Journal of the Mechanics and Physics of Solids, 48, (11), (2000), pp. 2419-2444. https://doi.org/10.1016/s00225096(00)00003-x.

[7] V. A. Marchenko and E. Y. Khruslov. Homogenization of partial differential equations, Vol. 46. Springer Science \& Business Media, (2008).

[8] U. Hornung. Homogenization and porous media, Vol. 6. Interdisciplinary Applied Mathematics, (1997).

[9] B. Gambin and E. Kröner. Higher-order terms in the homogenized stress-strain relation of periodic elastic media. Physica Status Solidi (B), 151, (2), (1989), pp. 513-519. https://doi.org/10.1002/pssb.2221510211.

[10] C. Boutin. Microstructural effects in elastic composites. International Journal of Solids and Structures, 33, (7), (1996), pp. 1023-105. https://doi.org/10.1016/0020-7683(95)00089-5.

[11] I. V. Andrianov, J. Awrejcewicz, and A. A. Diskovsky. Homogenization of quasiperiodic structures. Journal of Vibration and Acoustics, 128, (4), (2006), pp. 532-534. https://doi.org/10.1115/1.2202158.

[12] F. Su, Z. Xu, J. Z. Cui, and Q. L. Dong. Multi-scale method for the quasi-periodic structures of composite materials. Applied Mathematics and Computation, 217, (12), (2011), pp. 5847-5852. https://doi.org/10.1016/j.amc.2010.12.068.

[13] L. Guillot, Y. Capdeville, and J. J. Marigo. 2-D non-periodic homogenization of the elastic wave equation: SH case. Geophysical Journal International, 182, (3), (2010), pp. 1438-1454. https://doi.org/10.1111/j.1365-246x.2010.04688.x.

[14] Y. Capdeville, L. Guillot, and J. J. Marigo. 2-D non-periodic homogenization to upscale elastic media for P-SV waves. Geophysical Journal International, 182, (2), (2010), pp. 903-922. https://doi.org/10.1111/j.1365-246x.2010.04636.x.

[15] E. S. Palencia. Non-homogeneous media and vibration theory, Vol. 127. Springer-Verlag Berlin, (1980).

[16] F. Devries, H. Dumontet, G. Duvaut, and F. Léné. Homogenization and damage for composite structures. International Journal for Numerical Methods in Engineering, 27, (2), (1989), pp. 285-298. https://doi.org/10.1002/nme.1620270206.

[17] G. A. Francfort and J. J. Marigo. Stable damage evolution in a brittle continuous medium. European Journal of Mechanics Series a Solids, 12, (1993), pp. 149-149.

[18] K. Pham and J. J. Marigo. Approche variationnelle de l'endommagement: I. Les concepts fondamentaux. Comptes Rendus Mécanique, 338, (4), (2010), pp. 191-198. https://doi.org/10.1016/j.crme.2010.03.009.

[19] K. Pham and J. J. Marigo. Approche variationnelle de l'endommagement: II. Les modèles à gradient. Comptes Rendus Mécanique, 338, (4), (2010), pp. 199-206.

[20] C. Dascalu, G. Bilbie, and E. Agiasofitou. Damage and size effects in elastic solids: a homogenization approach. International Journal of Solids and Structures, 45, (2), (2008), pp. 409-430. https://doi.org/10.1016/j.ijsolstr.2007.08.025. 